\title{
SYNTHESIS, BIOLOGICAL AND INSILICO STUDIES OF SOME NEW N-(4-(BENZOFURAN-2-YL)-3-ARYLTHIAZOL-2- (3H)-YLIDENE)-ARYL AMINES AS POTENTIAL ANTI- CANCER AGENTS
}

\author{
Siddhartha Marupati ${ }^{1}$, Rajesh Kumar Gaddam ${ }^{2}$, B. Prasanna ${ }^{1, *}$ \\ and A. Thirupathaiah ${ }^{1}$ \\ ${ }^{1}$ Department of Chemistry, Chaitanya Postgraduate College (Autonomous), Kishanpura, \\ Hanamkonda, Warangal-506 001, Telangana, India \\ ${ }^{2}$ Department of Humanities and Sciences, Jawaharlal Nehru Technological University \\ Hyderabad College of Engineering Manthani (JNTUHCEM), \\ Peddapalli -505212, Telangana, India. \\ *E-mail: prasschem@gmail.com
}

\begin{abstract}
A series of some new $N$-(4-(benzofuran-2-yl)-3-arylthiazol-2(3H)-ylidene)-arylamines $\mathbf{4}(\mathbf{a}-\mathbf{q})$ has been synthesized from bromination of 2-acylbenzofuran than followed by condensation with aryl/heteroarylamines and arylthioisocyanates in ethanol. The synthesized compounds $\mathbf{4}(\mathbf{a}-\mathbf{q})$ screened for their antioxidant and anticancer activities. The data revealed that compounds $\mathbf{4 d}, \mathbf{4 l}, \mathbf{4 o}$ and $\mathbf{4 q}$ showed higher activity than the standard antioxidant ascorbic acid. The anticancer activity of the synthesized compound was also evaluated, compared to the standard drug doxorubicin against A549 and MDAMB-231 (Human Lung \& breast cancer cell lines). The data revealed that the compounds bearing, 4-chloro; 4-chloro (4a), 4-methoxy; 4-chloro (4d), 4-pyridinyl; 3-pyridine (4l), 4-chloro; 2,4-difluoro (4m) and 4-nitro; 4-nitro (4q) against A549 have shown significant activity with $\mathrm{IC}_{50}=8.00 \pm 1.21$ $\mu \mathrm{g} / \mathrm{mL}, \mathrm{IC}_{50}=4.12 \pm 1.51 \mu \mathrm{g} / \mathrm{mL}, \mathrm{IC}_{50}=6.21 \pm 0.37 \mu \mathrm{g} / \mathrm{mL}, 7.33 \pm 0.54 \mu \mathrm{g} / \mathrm{mL}$ and $\mathrm{IC}_{50}=7.80 \pm 0.57 \mu \mathrm{g} / \mathrm{mL}$ respectively. Similarly, compounds bearing, 4-methyl; phenyl (4e), 4-nitro; 3-chloro (4i), 4-pyridinyl; 3-pyridine (4l), 4-chloro; 3-trifluoro (4o) and 4-nitro; 4-nitro (4q) exhibited prominent activity against MDAMB-231 cell line with $\mathrm{IC}_{50}=4.11 \pm 0.59 \mu \mathrm{g} / \mathrm{mL}, \mathrm{IC}_{50}=2.43 \pm 1.12 \mu \mathrm{g} / \mathrm{mL}, \mathrm{IC}_{50}=3.80 \pm 0.52 \mu \mathrm{g} / \mathrm{mL}, \mathrm{IC}_{50}=3.73 \pm 0.35 \mu \mathrm{g} / \mathrm{mL}$ and $\mathrm{IC}_{50}=4.90 \pm 1.64 \mu \mathrm{g} / \mathrm{mL}$ respectively. Compounds $\mathbf{4 1}$ and $\mathbf{4 q}$ are promising compounds to sustain at low percentage of $\mathrm{IC}_{50}$ and revealed superior potency than doxorubicin, supported by antioxidant and molecular docking studies as well. Most of the newly synthesized compounds exhibit substantial activity in opposition to tested cell lines, and emerged as prospective drugs for further progress.
\end{abstract}

Keywords: Benzofuran, Antioxidant, anticancer activities, In-vitro and Molecular docking studies

(C) RASĀYAN. All rights reserved

\section{INTRODUCTION}

A balance between formation of "Reactive Oxygen Species," (ROS) and their detoxification is essential for normal cellular function. When such a balance is disrupted cell enters in a state of oxidative stress and is damaged, ${ }^{1}$ leads to a variety of disorders including oxidative damage to DNA, proteins, oxidative damage of tissues and biomolecules, a wide range of human diseases cancer, ${ }^{2}$ atherosclerosis, ${ }^{3}$ neurodegenerative disorders and aging ${ }^{4}$ in living beings. Antioxidants prevent oxidative damage induced by free radicals and ROS. These are capable of stabilizing or deactivating free radicals before they attack cells. Thus, antioxidant therapy has also gained immense importance in cancer therapy and in the treatment of several disorders. Cancer is continuing to be a leading cause of surpassing heart diseases. Lung cancer and malignant breast cancer (MBC) remain leading causes of cancer-related deaths in developing as well as undeveloped countries. Based on National Breast Cancer Foundation, it was probable that almost 200,000 women were diagnosed with breast cancer each year and more than 
40,000 will be die and about $90 \%$ of lung cancers are non-small cell lung cancer (NSCLC). The dysregulation of the epidermal growth factor receptor (EGFR) signaling pathway caused by genetic alterations in the kinase domain of EGFR is associated with malignant transformation in non-small cell lung cancer (NSCLC). ${ }^{5}$ Therefore, the EGFR family remains a major target for the development of anticancer agents. ${ }^{6}$ Aromatase, an enzyme that converts androgens to estrogens located in the endoplasmic reticulum of estrogen producing cells. MBC is functional, based on interfering with estrogen production or estrogen action or even both by lowering aromatase enzyme concentration in post-menopausal estrogen dependent breast cancer. ${ }^{7}$ Heterocyclic motifs belong to a special class of compounds which are used in medicinal chemistry ${ }^{8}$ for their improved properties like solubilities and salt formation, both of which are known to be important for oral absorption and bioavailability. ${ }^{9}$ In this context, oxygen heterocycles exhibit pharmacological activities. ${ }^{10}$ This has generated enormous interest in synthetic products containing benzofuran nucleus. Besides this, Benzofuran derivatives are reported as versatile biodynamic agents ${ }^{11}$ displaying a wide range of biological properties of which the most potent are antimicrobial, ${ }^{12}$ anti-inflammatory, analgesic and antipyretic, ${ }^{13}$ anticancer, ${ }^{14}$ antioxidant ${ }^{15}$ activities. In addition to this, thiazole is a crucial part with profound efficacy in vitamin-B1 (thiamine), penicillin, antibiotics such as micrococcin, troglitazone and possesses a wide range of biological activities like antimicrobial, ${ }^{13}$ antiinflammatory, ${ }^{14,15}$ anticancer, ${ }^{16}$ anti-HIV,${ }^{17}$ antioxidant, ${ }^{18}$ and analgesic. ${ }^{19}$

Inspired by the biological profile of benzofuran and thiazoles, in continuation of our ongoing research ${ }^{20-22}$ and interest in search for antimicrobials and anticancer drugs, we disclose the synthesis of a new library of hybrid benzofuran derivatives, $N$-(4-(benzofuran-2-yl)-3-arylthiazol-2(3H)-ylidene)arylamines $\mathbf{4}(\mathbf{a}-\mathbf{q}$ ) to enhance the biological activity. In the present study, antioxidant and anticancer studies are explored by varied substitution pattern on $2^{\text {nd }}$ and $3^{\text {rd }}$ positions of thiazole to vary electronic environment and elucidate the Structure-Activity Relationships (SAR) aiming at finding selective low toxic New Chemical Entities (NCEs), that preferably have chemical characteristics that clearly differ from those of existing agents and warrants for further drug development.

\section{EXPERIMENTAL}

All the reagents and solvents were commercially available and used without further purification unless otherwise stated. Melting points were uncorrected determined in open capillaries with a "Cintex" melting point apparatus. Elemental analysis was done by Carlo Erba EA 1108 analyzer. The purity of the compounds was checked by TLC plates purchased from Merck. IR spectra were recorded on a thermo Nicolet Nexus 670 Q8 instrument (KBr pellets). ${ }^{1} \mathrm{H}$ NMR spectra were recorded on a Bruker WM-400 spectrometer in $\delta$ ppm using TMS as an internal standard. Mass spectra (EI-MS) were determined on a Perkin Elmer spectrometer (SCIEX API-2000, ESI) at $12.5 \mathrm{eV}$.

\section{Synthesis of 2-bromoacetylbenzofuran (2)}

To a mixture of 2-acetylbenzofuran $1(50 \mathrm{mmol})$ in acetic acid $(50 \mathrm{~mL})$, bromine $(50 \mathrm{mmol})$ in acetic acid $(50 \mathrm{~mL})$ was added dropwise under nitrogen atmosphere and stirred for $90 \mathrm{~min}$ at $50{ }^{\circ} \mathrm{C}$. After completion of the reaction, cooled and added into crushed ice-water, solid separated and filtered. The solid was washed with water thrice and purified by recrystallizing from ethanol to get the pure compound 2 . Yield: $80 \%$; mp 88-90 ${ }^{\circ} \mathrm{C}$.

\section{General procedure for synthesis of $N$-(4-(benzofuran-2-yl)-3-(aryl/hetaryl)thiazol-2(3H)-ylide- ne)arylamines $4(\mathbf{a}-\mathbf{q})$}

A mixture of 2-(2-bromoacetyl)benzofuran 2 (1 mmol), aryl/hetaryl primary amine $(1 \mathrm{mmol})$ and phenyl isothiocyanate $\mathbf{3}(1 \mathrm{mmol})$ were taken in absolute ethanol $(5 \mathrm{ml})$ and refluxed for about 30 minutes. After completed the reaction, the reaction mixture was cooled, solid separated, filtered then the compound was purified by re-crystallizing from ethanol.

$\mathrm{N}$-(4-(benzofuran-2-yl)-3-(4-chlorophenyl)thiazol-2(3H)-ylidene)-4-chloro benzene amine (4a) Off white solid; yield: 82\%; mp: 140-142 ${ }^{\circ} \mathrm{C}$. IR (KBr): 3051, $3043(\mathrm{Ar}-\mathrm{H}), 1622(\mathrm{C}=\mathrm{N}), 1599(\mathrm{C}=\mathrm{C})$, 1283 (C-S), 1081 (C-O), $752(\mathrm{C}-\mathrm{Cl}) \mathrm{cm}^{-1} .{ }^{1} \mathbf{H}-\mathbf{N M R}$ (400 MHz, DMSO-d $)_{6}: \delta$ 7.63-7.61 (m, 2H, Ar-H), 
7.57-7.51 (m, 3H, Ar-H), 7.49 (m, 1H, Ar-H), 7.36-7.29 (m, 3H, Ar-H), 7.22-7.19 (m, 1H, Ar-H), 7.06 $(\mathrm{m}, 1 \mathrm{H}, \mathrm{Ar}-\mathrm{H}), 6.95-6.93(\mathrm{~m}, 2 \mathrm{H}, \mathrm{Ar}-\mathrm{H}), 5.91(\mathrm{~s}, 1 \mathrm{H}, \mathrm{Ar}-\mathrm{H}) .{ }^{13} \mathrm{C}$ NMR $\left(100 \mathrm{MHz}, \mathrm{DMSO}-d_{6}\right): \delta 161.2$ (C, C-2), 158.1 (C, C-2'), 154.4 (C, C-7a'), 152.0 (C, C-4), 146.2 (C, C-1"'), 139.6 (C, C-1"'), 133.0 (C, C$\left.4^{\prime \prime \prime}\right), 132.9$ (C, C-3a'), 131.0 (CH, C-3"', CH, C-5"'), 128.2 (CH, C-3", CH, C-5"'), 126.1 (C, C-4"), 125.2 (C, C-6'), $124.5\left(\mathrm{CH}, \mathrm{C}-2^{\prime \prime \prime}, \mathrm{CH}, \mathrm{C}-6^{\prime \prime \prime}\right), 123.2,122.8\left(\mathrm{CH}, \mathrm{C}-5^{\prime}\right), 120.2\left(\mathrm{CH}, \mathrm{C}-4^{\prime}\right), 119.1\left(\mathrm{CH}, \mathrm{C}-2^{\prime \prime}, \mathrm{CH}\right.$, C-6"), $114.1(\mathrm{CH}, \mathrm{C}-5), 111.4(\mathrm{CH}, \mathrm{C}-7), 108.2\left(\mathrm{CH}, \mathrm{C}-3^{\prime}\right)$. MS m/z: $437\left(\mathrm{M}^{+}+1\right)$. Anal. Calcd. for $\mathrm{C}_{23} \mathrm{H}_{14} \mathrm{Cl}_{2} \mathrm{~N}_{2} \mathrm{OS}$ : C, 63.16; H, 3.23; N, 6.41. Found: C, 63.13; H, 3.20; N, 6.38.

\section{$\mathrm{N}$-(4-(benzofuran-2-yl)-3-p-tolylthiazol-2(3H)-ylidene)-4-chlorobenzenamine (4b)}

Light yellow solid; yield: 78\%; mp: 152-155 ${ }^{\circ} \mathrm{C}$. IR (KBr): 3055, $3047(\mathrm{Ar}-\mathrm{H}), 2971(\mathrm{C}-\mathrm{H}), 1620(\mathrm{C}=\mathrm{N})$, $1603(\mathrm{C}=\mathrm{C}), 1283(\mathrm{C}-\mathrm{S}), 1088(\mathrm{C}-\mathrm{O}), 733(\mathrm{C}-\mathrm{Cl}) \mathrm{cm}^{-1} .{ }^{1} \mathbf{H}-\mathrm{NMR}\left(400 \mathrm{MHz}, \mathrm{DMSO}-d_{6}\right): \delta$ 7.86-7.79 (m, $1 \mathrm{H}, \mathrm{ArH}), 7.55-7.46(\mathrm{~m}, 5 \mathrm{H}, \mathrm{ArH}), 7.36-7.32(\mathrm{~m}, 3 \mathrm{H}, \mathrm{ArH}), 7.24-7.20(\mathrm{~m}, 4 \mathrm{H}, \mathrm{ArH}), 5.74(\mathrm{~s}, 1 \mathrm{H}, \mathrm{ArH})$, $2.45\left(\mathrm{~s}, 3 \mathrm{H}, \mathrm{CH}_{3}\right) .{ }^{13} \mathrm{C}$ NMR $\left(100 \mathrm{MHz}, \mathrm{DMSO}-d_{6}\right): \delta 161.2(\mathrm{C}, \mathrm{C}-2), 158.2\left(\mathrm{C}, \mathrm{C}-2^{\prime}\right), 154.3\left(\mathrm{C}, \mathrm{C}-7 \mathrm{a}^{\prime}\right)$, 152.0 (C, C-4), 148.2 (C, C-4"'), 140.1 (C, C-1"'), 133.4 (C, C-1"'), 132.7 (C, C-3a'), 130.4 (CH, C-3", $\left.\mathrm{CH}, \mathrm{C}-5^{\prime \prime}\right), 129.1\left(\mathrm{C}, \mathrm{C}-4^{\prime \prime}\right), 127.1\left(\mathrm{CH}, \mathrm{C}-2^{\prime \prime \prime}, \mathrm{CH}, \mathrm{C}-6^{\prime \prime \prime}\right), 126.1$ (C, C-6'), $124.2\left(\mathrm{CH}, \mathrm{C}-3^{\prime \prime \prime}, \mathrm{CH}, \mathrm{C}-5^{\prime \prime \prime}\right)$, $122.4\left(\mathrm{CH}, \mathrm{C}-5^{\prime}\right), 120.1\left(\mathrm{CH}, \mathrm{C}-4^{\prime}\right), 117.1\left(\mathrm{CH}, \mathrm{C}-2^{\prime \prime}, \mathrm{CH}, \mathrm{C}-6^{\prime \prime}\right), 114.3(\mathrm{CH}, \mathrm{C}-5), 111.6(\mathrm{CH}, \mathrm{C}-7)$, $108.3\left(\mathrm{CH}, \mathrm{C}-3^{\prime}\right), 22.6\left(\mathrm{CH}_{3}, \mathrm{C}-1^{\prime \prime \prime \prime}\right)$. MS m/z: $417\left(\mathrm{M}^{+}+1\right)$. Anal. Calcd. for $\mathrm{C}_{24} \mathrm{H}_{17} \mathrm{ClN}_{2} \mathrm{OS}$ : C, 69.14; H, 4.11; N, 6.72. Found: C, 69.12; H, 4.07; N, 6.68.

\section{$\mathrm{N}$-(4-(benzofuran-2-yl)-3-(4-chlorophenyl)thiazol-2(3H)-ylidene)benzenamine (4c)}

White solid; yield $81 \%$; mp 136-138 ${ }^{\circ} \mathrm{C}$. IR (KBr): 3040, $3032(\mathrm{Ar}-\mathrm{H}), 1595(\mathrm{C}=\mathrm{N}), 1605(\mathrm{C}=\mathrm{C}), 1279$ (C-S), 1078 (C-O), $759(\mathrm{C}-\mathrm{Cl}) \mathrm{cm}^{-1}$. ${ }^{1} \mathrm{H}-\mathrm{NMR}$ (400 MHz, DMSO-d $)$ : $\delta$ 7.58-7.46 (m, 7H, ArH), 7.35$7.30(\mathrm{~m}, 2 \mathrm{H}, \mathrm{ArH}), 7.20(\mathrm{~m}, 1 \mathrm{H}, \mathrm{ArH}), 7.18-7.17(\mathrm{~m}, 1 \mathrm{H}, \mathrm{ArH}), 7.05(\mathrm{~m}, 1 \mathrm{H}, \mathrm{ArH})$, 6.94-6.92 (m, 2H, $\mathrm{ArH}), 5.65$ (s, 1H, ArH). ${ }^{13} \mathrm{C}$ NMR (100 MHz, DMSO-d $)$ : $\delta 161.1$ (C, C-2), 158.3 (C, C-2'), 154.3 (C, C-7a'), 152.2 (C, C-4), 149.4 (C, C-1"'), 140.2 (C, C-1"), 132.7 (C, C-3a'), 131.8 (CH, C-3"', CH, C-5"'), $130.1\left(\mathrm{CH}, \mathrm{C}-3^{\prime \prime}, \mathrm{CH}, \mathrm{C}-5^{\prime \prime}\right), 128.1$ (CH, C-4"'), 126.3 (C, C-6', C, C-4"'), 124.6 (CH, C-2'"', CH, C-6"'), $122.1\left(\mathrm{CH}, \mathrm{C}-2^{\prime \prime}, \mathrm{CH}, \mathrm{C}-6^{\prime \prime}\right), 122.9\left(\mathrm{CH}, \mathrm{C}-5^{\prime}\right), 120.4\left(\mathrm{CH}, \mathrm{C}-4^{\prime}\right), 114.2(\mathrm{CH}, \mathrm{C}-5), 111.6(\mathrm{CH}, \mathrm{C}-7)$, 108.1 (CH, C-3'). MS m/z: $403\left(\mathrm{M}^{+}+1\right)$. Anal. Calcd. for $\mathrm{C}_{23} \mathrm{H}_{15} \mathrm{ClN}_{2} \mathrm{OS}$ : C, 68.57; H, 3.75; N, 6.95. Found: C, 68.55; H, 3.72; N, 6.93 .

$\mathrm{N}$-(4-(benzofuran-2-yl)-3-(4-methoxyphenyl)thiazol-2(3H)-ylidene)-4-chloro benzene amine (4d) Off white solid; yield: $79 \%$; mp: 161-164 ${ }^{\circ} \mathrm{C}$. IR (KBr): 3048, $3031(\mathrm{Ar}-\mathrm{H}), 1610(\mathrm{C}=\mathrm{N}), 1592(\mathrm{C}=\mathrm{C})$, 1217 (C-O), 1283 (C-S), 1082 (C-O), $748(\mathrm{C}-\mathrm{Cl}) \mathrm{cm}^{-1}$. ${ }^{1} \mathbf{H}-\mathrm{NMR}$ (400 MHz, DMSO-d $)$ : $\delta$ 7.64-7.62 (m, $2 \mathrm{H}, \mathrm{ArH}), 7.56-7.50(\mathrm{~m}, 4 \mathrm{H}, \mathrm{ArH}), 7.39-7.33(\mathrm{~m}, 2 \mathrm{H}, \mathrm{ArH}), 7.28-7.21(\mathrm{~m}, 5 \mathrm{H}, \mathrm{ArH}), 5.75(\mathrm{~m}, 1 \mathrm{H}, \mathrm{ArH})$, $3.88\left(\mathrm{~s}, 3 \mathrm{H}, \mathrm{OCH}_{3}\right) .{ }^{13} \mathrm{C}$ NMR $\left(100 \mathrm{MHz}, \mathrm{DMSO}-d_{6}\right): \delta 161.4(\mathrm{C}, \mathrm{C}-2), 158.2\left(\mathrm{C}, \mathrm{C}-2^{\prime}\right), 154.3(\mathrm{C}, \mathrm{C}-$ 7a'), 152.2 (C, C-4), 150.2 (C, C-4"), 148.1 (C, C-1"'), 135.2 (C, C-1'"'), 133.1 (C, C-4"'), 132.8 (C, C3a'), 130.8 (CH, C-3"', CH, C-5"'), 124.6 (C, C-6', CH, C-2'"', CH, C-6"'), $122.8\left(\mathrm{CH}, \mathrm{C}-5^{\prime}\right), 121.5(\mathrm{CH}$, C-2", CH, C-6"), 120.4 (CH, C-4'), 118.1 (CH, C-3"', CH, C-5"'), 114.3 (CH, C-5), 111.5 (CH, C-7), $108.2\left(\mathrm{CH}, \mathrm{C}-3^{\prime}\right), 54.3$ (C, C-1"'"'). MS m/z: $432\left(\mathrm{M}^{+}+1\right)$. Anal. Calcd. for $\mathrm{C}_{24} \mathrm{H}_{17} \mathrm{ClN}_{2} \mathrm{O}_{2} \mathrm{~S}: \mathrm{C}, 66.58 ; \mathrm{H}$, 3.96; N, 6.47. Found: C, 66.54; H, 3.93; N, 6.44.

$\mathrm{N}$-(4-(benzofuran-2-yl)-3-p-tolylthiazol-2(3H)-ylidene)benzenamine (4e)

Light Yellow solid; yield: $76 \%$; mp 144-146 ${ }^{\circ} \mathrm{C}$. IR (KBr): 3052, $3041(\mathrm{Ar}-\mathrm{H}), 2961(\mathrm{C}-\mathrm{H}), 1624(\mathrm{C}=\mathrm{N})$, $1604(\mathrm{C}=\mathrm{C}), 1278(\mathrm{C}-\mathrm{S}), 1079(\mathrm{C}-\mathrm{O}), 752(\mathrm{C}-\mathrm{Cl}) \mathrm{cm}^{-1} .{ }^{1} \mathbf{H}-\mathrm{NMR}\left(400 \mathrm{MHz}, \mathrm{DMSO}-d_{6}\right)$ : $\delta$ 7.74-7.73 (m, 2H, ArH), 7.64-7.61 (m, 6H, ArH), 7.54-7.52 (m, 2H, ArH), 7.24-7.20 (m, 4H, ArH), $5.92(\mathrm{~s}, 1 \mathrm{H}, \mathrm{ArH})$, $2.46\left(\mathrm{~s}, 3 \mathrm{H}, \mathrm{CH}_{3}\right) .{ }^{13} \mathrm{C}$ NMR $\left(100 \mathrm{MHz}, \mathrm{DMSO}-d_{6}\right): \delta 161.4(\mathrm{C}, \mathrm{C}-2), 158.2\left(\mathrm{C}, \mathrm{C}-2^{\prime}\right), 154.6\left(\mathrm{C}, \mathrm{C}-7 \mathrm{a}^{\prime}\right)$, 152.2 (C, C-4), 150.1 (C, C-1"'), 139.0 (C, C-1"), 132.8 (C, C-3a'), 132.4 (C, C-4"), 131.7 (CH, C-3"', CH, C-5"'), 130.4 (CH, C-2", CH, C-6"'), 129.4 (C, C-4"'), 124.6 (C, C-6'), 123.1 (CH, C-2"', CH, C-6"'), $122.7\left(\mathrm{CH}, \mathrm{C}-5^{\prime}\right), 120.3\left(\mathrm{CH}, \mathrm{C}-4^{\prime}\right), 117.0\left(\mathrm{CH}, \mathrm{C}-3^{\prime \prime}, \mathrm{CH}, \mathrm{C}-5^{\prime \prime}\right), 114.3(\mathrm{CH}, \mathrm{C}-5), 111.5(\mathrm{CH}, \mathrm{C}-7)$, $108.0\left(\mathrm{CH}, \mathrm{C}-3^{\prime}\right), 23.4$ (C, C-1"'"'). MS m/z: $382\left(\mathrm{M}^{+}\right)$. Anal. Calcd. for $\mathrm{C}_{24} \mathrm{H}_{18} \mathrm{~N}_{2} \mathrm{OS}: \mathrm{C}, 75.37 ; \mathrm{H}, 4.74$; N, 7.32. Found: C, 75.33; H, 4.71; N, 7.29. 
RASĀYAN J. Chem.

Vol. 11 | No. 2 |661 - 675 | April - June | 2018

$\mathrm{N}$-(3-((1H-indol-2-yl)methyl)-4-(benzofuran-2-yl)thiazol-2(3H)-ylidene)benzenamine (4f)

White solid; yield: 78 \%; mp: 120-123 ${ }^{\circ} \mathrm{C}$. IR (KBr): $3192(\mathrm{~N}-\mathrm{H})$, 3048, $3032(\mathrm{Ar}-\mathrm{H}), 1616(\mathrm{C}=\mathrm{N}), 1595$ $(\mathrm{C}=\mathrm{C}), 1276(\mathrm{C}-\mathrm{S}), 1082(\mathrm{C}-\mathrm{O}), 746(\mathrm{C}-\mathrm{Cl}) \mathrm{cm}^{-1} .{ }^{1}$ H-NMR (400 MHz, DMSO-d $\left.)_{6}\right): \delta 10.89(\mathrm{~s}, 1 \mathrm{H}, \mathrm{NH})$, 7.61-7.52 (m, 4H, ArH), 7.40-7.32 (m, 4H, ArH), 7.21-7.18 (m, 4H, ArH), 7.06-7.03 (m, 2H, ArH), 6.85 $(\mathrm{m}, 2 \mathrm{H}, \mathrm{ArH}), 3.88\left(\mathrm{~s}, 2 \mathrm{H}, \mathrm{CH}_{2}\right) .{ }^{13} \mathrm{C}$ NMR $\left(100 \mathrm{MHz}, \mathrm{DMSO}-d_{6}\right): \delta 161.3(\mathrm{C}, \mathrm{C}-2), 158.2\left(\mathrm{C}, \mathrm{C}-2^{\prime}\right)$, $154.5\left(\mathrm{C}, \mathrm{C}-7 \mathrm{a}^{\prime}\right), 152.2$ (C, C-4), 150.3 (C, C-1"'), 141.0 (C, C-7a'), 138.1 (C, C-2"'), 132.6 (C, C-3a'), 131.6 (CH, C-3"', CH, C-5"'), 130.0 (CH, C-4"'), 129.2 (C, C-3a"'), 126.2 (C, C-6'), 124.4 (C, C-6"), 122.8 (CH, C-5', CH, C-2'"', CH, C-6"' ), 121.6 (CH, C-5"), 120.5 (CH, C-4', CH, C-7"'), 114.2 (CH, C-5), $112.0\left(\mathrm{CH}, \mathrm{C}-4^{\prime \prime}\right), 111.3(\mathrm{CH}, \mathrm{C}-7), 108.1\left(\mathrm{CH}, \mathrm{C}-3^{\prime}\right), 106.0\left(\mathrm{CH}, \mathrm{C}-3^{\prime \prime}\right), 46.2\left(\mathrm{CH}_{2}, \mathrm{C}-1^{\prime \prime \prime \prime}\right)$. MS m/z: 421 $\left(\mathrm{M}^{+}\right)$. Anal. Calcd. for $\mathrm{C}_{26} \mathrm{H}_{19} \mathrm{~N}_{3} \mathrm{OS}$ : C, 74.08; H, 4.54; N, 9.97. Found: C, 74.04; H, 4.51; N, 9.94 .

$\mathrm{N}$-(4-(benzofuran-2-yl)-3-(4-methoxyphenyl)thiazol-2(3H)-ylidene)-3-chloro benzene amine (4g) Off white solid; yield: $75 \%$; mp 151-152 ${ }^{\circ} \mathrm{C}$. IR (KBr): 3055, $3043(\mathrm{Ar}-\mathrm{H}), 1610(\mathrm{C}=\mathrm{N}), 1592(\mathrm{C}=\mathrm{C})$, 1258 (C-S), 1223 (C-O), 1092 (C-O-C), $754(\mathrm{C}-\mathrm{Cl}) \mathrm{cm}^{-1} .{ }^{1} \mathbf{H}-\mathrm{NMR}$ (400 MHz, DMSO-d 6 ): $\delta$ 7.82-7.79 $(\mathrm{m}, 2 \mathrm{H}, \mathrm{ArH}), 7.53-7.45$ (m, 6H, ArH), 7.26-7.18 (m, 3H, ArH), 6.88 (m, 2H, ArH), 5.74 (s, 1H, ArH), $3.86\left(\mathrm{~s}, 3 \mathrm{H}, \mathrm{OCH}_{3}\right) .{ }^{13} \mathrm{C}$ NMR $\left(100 \mathrm{MHz}, \mathrm{DMSO}-d_{6}\right): \delta 161.3(\mathrm{C}, \mathrm{C}-2), 158.2\left(\mathrm{C}, \mathrm{C}-2^{\prime}\right), 154.5\left(\mathrm{C}, \mathrm{C}-7 \mathrm{a}^{\prime}\right)$, 152.1 (C, C-4), 151.2 (C, C-1"'), 150.0 (C, C-4"), 136.0 (C, C-3"'), 134.0 (C, C-1"'), 133.0 (C, C-5"'), 132.7 (C, C-3a'), 128.1 (C, C-4"'), 125.2 (C, C-2'"'), 124.4 (C, C-6'), 122.6 (CH, C-5'), 121.4 (C, C-6"'), $120.4\left(\mathrm{CH}, \mathrm{C}-4^{\prime}\right), 117.8$ (CH, C-2", CH, C-6"), 115.3 (CH, C-3", CH, C-5"), $114.3(\mathrm{CH}, \mathrm{C}-5), 111.5(\mathrm{CH}$, C-7), 108.1 (CH, C-3'), $55.1\left(\mathrm{CH}_{3}, \mathrm{C}-1^{\prime \prime \prime \prime}\right)$. MS m/z: $432\left(\mathrm{M}^{+}\right)$. Anal. Calcd. for $\mathrm{C}_{24} \mathrm{H}_{17} \mathrm{ClN}_{2} \mathrm{O}_{2} \mathrm{~S}: \mathrm{C}$, 66.58; H, 3.96; N, 6.47. Found: C, 66.55; H, 3.93; N, 6.43.

\section{$\mathrm{N}$-(4-(benzofuran-2-yl)-3-p-tolylthiazol-2(3H)-ylidene)-3-chlorobenzenamine (4h)}

Yellow solid; yield: $74 \%$; mp 136-138 ${ }^{\circ} \mathrm{C}$. IR (KBr): 3065, $3041(\mathrm{Ar}-\mathrm{H}), 2893(\mathrm{C}-\mathrm{H}), 1620(\mathrm{C}=\mathrm{N}), 1596$ $(\mathrm{C}=\mathrm{C}), 1262(\mathrm{C}-\mathrm{S}), 1088(\mathrm{C}-\mathrm{O}), 749(\mathrm{C}-\mathrm{Cl}) \mathrm{cm}^{-1} .{ }^{1} \mathbf{H}-\mathrm{NMR}\left(400 \mathrm{MHz}, \mathrm{DMSO}-d_{6}\right): \delta$ 7.76-7.69 (m, 4H, ArH), 7.54-7.48 (m, 4H, ArH), 7.26-7.22 (m, 4H, ArH), 6.91 (m, 1H, ArH), 5.76 (s, 1H, ArH), $3.86(\mathrm{~s}$, $\left.3 \mathrm{H}, \mathrm{OCH}_{3}\right) .{ }^{13} \mathrm{C}$ NMR $\left(100 \mathrm{MHz}, \mathrm{DMSO}-d_{6}\right): \delta 161.1(\mathrm{C}, \mathrm{C}-2), 158.3\left(\mathrm{C}, \mathrm{C}-2^{\prime}\right), 154.6\left(\mathrm{C}, \mathrm{C}-7 \mathrm{a}^{\prime}\right), 151.8$ (C, C-4), 150.6 (C, C-1"'), 139.3 (C, C-1"), 135.8 (C, C-3"'), 132.5 (C, C-3a'), 133.4 (C, C-5'"'), 130.4 $\left(\mathrm{CH}, \mathrm{C}-3^{\prime \prime}, \mathrm{CH}, \mathrm{C}-5^{\prime \prime}\right), 129.1$ (C, C-4"), $128.1\left(\mathrm{CH}, \mathrm{C}-4^{\prime \prime \prime}\right), 124.8$ (C, C-6'), $123.7\left(\mathrm{CH}, \mathrm{C}-2^{\prime \prime \prime}\right), 122.3(\mathrm{CH}$, C-5') $118.9\left(\mathrm{CH}, \mathrm{C}-2^{\prime \prime}, \mathrm{CH}, \mathrm{C}-6^{\prime \prime}\right), 120.6\left(\mathrm{CH}, \mathrm{C}-4^{\prime}, \mathrm{CH}, \mathrm{C}-6^{\prime \prime \prime}\right), 111.2\left(\mathrm{CH}, \mathrm{C}-7^{\prime}\right), 108.1\left(\mathrm{CH}, \mathrm{C}-3^{\prime}\right), 22.2$ $\left(\mathrm{CH}_{3}, \mathrm{C}-1^{\prime \prime \prime \prime}\right)$. MS m/z: $416\left(\mathrm{M}^{+}\right)$. Anal. Calcd. for $\mathrm{C}_{24} \mathrm{H}_{17} \mathrm{ClN}_{2} \mathrm{OS}: \mathrm{C}, 69.14 ; \mathrm{H}, 4.11 ; \mathrm{N}, 6.72$. Found: $\mathrm{C}$, $69.10 ; \mathrm{H}, 4.09 ; \mathrm{N}, 6.68$.

$\mathrm{N}$-(4-(benzofuran-2-yl)-3-(4-nitrophenyl)thiazol-2(3H)-ylidene)-3-chlorobenzenamine (4i)

Pale orange solid; yield: $72 \%$; mp 144-146 ${ }^{\circ} \mathrm{C}$. IR (KBr): 3049, $3031(\mathrm{Ar}-\mathrm{H}), 1613(\mathrm{C}=\mathrm{N}), 1593(\mathrm{C}=\mathrm{C})$, 1528, $1357\left(\mathrm{NO}_{2}\right), 1269(\mathrm{C}-\mathrm{S}), 1093(\mathrm{C}-\mathrm{O}), 752(\mathrm{C}-\mathrm{Cl}) \mathrm{cm}^{-1} .{ }^{1} \mathbf{H}-\mathrm{NMR}$ (400 MHz, DMSO-d $)$ : $\delta 8.10(\mathrm{~d}$, $J=8.8 \mathrm{~Hz}, 2 \mathrm{H}, \mathrm{ArH}), 7.72-7.66(\mathrm{~m}, 4 \mathrm{H}, \mathrm{ArH}), 7.58-7.52(\mathrm{~m}, 4 \mathrm{H}, \mathrm{ArH}), 7.12(\mathrm{~d}, J=8.8 \mathrm{~Hz}, 2 \mathrm{H}, \operatorname{ArH})$, $7.02(\mathrm{~m}, 1 \mathrm{H}, \mathrm{ArH}), 5.85$ (s, 1H, ArH). ${ }^{13} \mathrm{C} \mathrm{NMR}\left(100 \mathrm{MHz}, \mathrm{DMSO}-d_{6}\right): \delta 161.1$ (C, C-2), $158.3(\mathrm{C}, \mathrm{C}-$ 2'), 154.6 (C, C-7a'), 151.8 (C, C-4), 150.6 (C, C-1"'), 139.3 (C, C-4"), 135.8 (C, C-3"'), 132.5 (C, C-3a'), 133.4 (C, C-5"'), 122.8 (CH, C-3", CH, C-5"), $119.1\left(\mathrm{CH}, \mathrm{C}-2^{\prime \prime}, \mathrm{CH}, \mathrm{C}-6^{\prime \prime}\right), 128.1\left(\mathrm{CH}, \mathrm{C}-4^{\prime \prime \prime}\right), 124.8(\mathrm{C}$, C-6'), 123.7 (CH, C-2"'), 122.3 (CH, C-5'), 146.9 (C, C-4"), 120.6 (CH, C-4', CH, C-6"') 114.3 (CH, C5), $111.2(\mathrm{CH}, \mathrm{C}-7), 108.1\left(\mathrm{CH}, \mathrm{C}-3^{\prime}\right)$. MS m/z: $447\left(\mathrm{M}^{+}\right)$. Anal. Calcd. for $\mathrm{C}_{23} \mathrm{H}_{14} \mathrm{ClN}_{3} \mathrm{O}_{3} \mathrm{~S}: \mathrm{C}, 61.68 ; \mathrm{H}$, 3.15 ; N, 9.38; Found: C, 61.63; H, 3.12; N, 9.34;

$\mathrm{N}$-(4-(benzofuran-2-yl)-3-(4-pyridyl)thiazol-2(3H)-ylidene)-3-chlorobenzenamine (4j)

Brown solid; yield $68 \%$; mp 138-141 ${ }^{\circ} \mathrm{C}$. IR (KBr): 3054, $3042(\mathrm{Ar}-\mathrm{H}), 1620(\mathrm{C}=\mathrm{N}), 1601(\mathrm{C}=\mathrm{C}), 1274$ (C-S), 1085 (C-O-C), 752 (C-Cl) cm ${ }^{-1} .{ }^{1} \mathrm{H}-\mathrm{NMR}$ (400 MHz, DMSO-d $\left.d_{6}\right): \delta 8.05$ (d, $\left.J=5.9 \mathrm{~Hz}, 2 \mathrm{H}, \mathrm{ArH}\right)$, 7.73-7.64 (m, 4H, ArH), 7.56-7.49 (m, 4H, ArH), 7.36 (d, J=5.9 Hz, 2H, ArH), 6.93 (m, 1H, ArH), 5.83 (s, $1 \mathrm{H}, \mathrm{ArH}) .{ }^{13} \mathrm{C}$ NMR (100 MHz, DMSO- $\left.d_{6}\right): \delta 161.1$ (C, C-2), 158.3 (C, C-2'), 156.0 (C, C-4"), 154.6 (C, C-7a'), 151.8 (C, C-4), $152.1\left(\mathrm{CH}, \mathrm{C}-2^{\prime \prime}, \mathrm{CH}, \mathrm{C}-6^{\prime \prime}\right), 150.6\left(\mathrm{C}, \mathrm{C}-1^{\prime \prime \prime}\right), 139.3\left(\mathrm{C}, \mathrm{C}-1^{\prime \prime}\right), 135.8$ (C, C3"'), 132.5 (C, C-3a'), 133.4 (C, C-5'"'), 112.0 (CH, C-3", CH, C-5"), 128.1 (CH, C-4"'), 124.8 (C, C-6'), 
123.7 (CH, C-2"'), 122.3 (CH, C-5'), 120.6 (CH, C-4', CH, C-6"'), 114.3 (CH, C-5), 111.2 (CH, C-7), $108.1\left(\mathrm{CH}, \mathrm{C}-3^{\prime}\right)$. MS m/z: $403\left(\mathrm{M}^{+}\right)$. Anal. Calcd. for $\mathrm{C}_{22} \mathrm{H}_{14} \mathrm{ClN}_{3} \mathrm{OS}: \mathrm{C}, 65.42 ; \mathrm{H}, 3.49 ; \mathrm{N}, 10.40$; Found: C, 65.38; H, 3.42; N, 10.35 .

N-(3-((1H-indol-2-yl)methyl)-4-(benzofuran-2-yl)thiazol-2(3H)-ylidene)-3-pyridyl amine (4k) Off white solid; yield $64 \%$; mp: 144-146 ${ }^{\circ} \mathrm{C}$. IR (KBr): $3214(\mathrm{~N}-\mathrm{H}), 3050,3038(\mathrm{Ar}-\mathrm{H}), 1613(\mathrm{C}=\mathrm{N})$, $1603(\mathrm{C}=\mathrm{C}), 1262(\mathrm{C}-\mathrm{S}), 1079(\mathrm{C}-\mathrm{O}-\mathrm{C}), 746(\mathrm{C}-\mathrm{Cl}) \mathrm{cm}^{-1}{ }^{1}{ }^{\mathbf{H}} \mathrm{H}-\mathrm{NMR}$ (400 MHz, DMSO-d $): \delta 10.84(\mathrm{~s}$, $1 \mathrm{H}, \mathrm{NH}), 9.09$ (s, 1H, ArH), $8.64(\mathrm{~d}, J=5 \mathrm{~Hz}, 1 \mathrm{H}, \mathrm{ArH}), 7.96(\mathrm{~d}, J=5 \mathrm{~Hz}, 1 \mathrm{H}, \mathrm{ArH}), 7.82(\mathrm{dd}, J=8.2$ and 5.2 Hz, 1H, ArH), 7.71-7.63 (m, 4H, ArH), 7.46-7.35 (m, 4H, ArH), $6.98(\mathrm{~m}, 1 \mathrm{H}, \mathrm{ArH}), 6.28(\mathrm{~s}, 1 \mathrm{H}$, $\mathrm{ArH}), 5.82(\mathrm{~s}, 1 \mathrm{H}, \mathrm{ArH}), 3.82\left(\mathrm{~s}, 2 \mathrm{H}, \mathrm{CH}_{2}\right) .{ }^{13} \mathrm{C}$ NMR (100 MHz, DMSO- $\left.d_{6}\right): \delta 161.1(\mathrm{C}, \mathrm{C}-2), 158.3(\mathrm{C}$, C-2'), 154.6 (C, C-7a'), 152.8 (C, C-4, C-6"'), 150.6 (C, C-2"' '), 141.0 (C, C-7a"), 138.1 (C, C-2"), 136.7 (C, C-3"'), 132.5 (C, C-3a'), 131.2 (C, C-4"'), 129.2 (C, C-3a"'), 128.5 (CH, C-5"'), 124.6 (C, C-6', C-6"), $122.3\left(\mathrm{CH}, \mathrm{C}-5^{\prime}, \mathrm{CH}, \mathrm{C}-7^{\prime}\right), 121.6\left(\mathrm{CH}, \mathrm{C}-5^{\prime \prime}\right), 120.6\left(\mathrm{CH}, \mathrm{C}-4^{\prime}\right), 114.3(\mathrm{CH}, \mathrm{C}-5), 112.0\left(\mathrm{CH}, \mathrm{C}-4^{\prime \prime}\right)$, 111.2 (CH, C-7"), 108.1 (CH, C-3'), 106.0 (CH, C-3"), $46.2\left(\mathrm{CH}_{2}, \mathrm{C}-1^{\prime \prime \prime \prime}\right)$. MS m/z: 422 (M+). Anal. Calcd. for $\mathrm{C}_{25} \mathrm{H}_{18} \mathrm{~N}_{4} \mathrm{OS}$ : C, 71.07; H, 4.29; N, 13.26; Found: C, 70.96; H, 4.25; N, 13.23.

$\mathrm{N}$-(4-(benzofuran-2-yl)-3-(4-pyridyl)thiazol-2(3H)-ylidene)-3-pyridylamine (4I)

Pale brown solid; yield: $76 \%$; mp: $151-153{ }^{\circ} \mathrm{C}$. IR (KBr): 3046, $3034(\mathrm{Ar}-\mathrm{H}), 1627(\mathrm{C}=\mathrm{N}), 1593(\mathrm{C}=\mathrm{C})$, 1271 (C-S), 1082 (C-O-C), 749 (C-Cl) cm ${ }^{-1} .{ }^{1} \mathbf{H}-N M R ~\left(400 \mathrm{MHz}, \mathrm{DMSO}-d_{6}\right): \delta 9.09$ (s, 1H, ArH, Pyridine, H-2), 8.64 (d, $J=5.2 \mathrm{~Hz}, 1 \mathrm{H}, \mathrm{ArH}$, Pyridine, H-6), 8.05 (d, $J=5.9 \mathrm{~Hz}, 2 \mathrm{H}, \mathrm{ArH}, 4$-Pyridine, H2, 6), 7.96 (d, $J=5.2 \mathrm{~Hz}, 1 \mathrm{H}$, ArH, Pyridine, H-4), 7.82 (dd, $J=8.3$ and $5.2 \mathrm{~Hz}, 1 \mathrm{H}$, ArH, Pyridine, H-5), 7.76-7.64 (m, 4H, ArH, benzofuran), $7.36(\mathrm{~d}, J=5.9 \mathrm{~Hz}, 2 \mathrm{H}, \mathrm{ArH}, 4$-Pyridine, $\mathrm{H}-3,5), 6.96(\mathrm{~m}, 1 \mathrm{H}$, ArH, benzofuran), 5.88 (s, $1 \mathrm{H}, \mathrm{ArH}$, Thiazole). ${ }^{13} \mathrm{C}$ NMR (100 MHz, DMSO- $\left.d_{6}\right): \delta 161.1$ (C, C-2), 158.3 (C, C-2'), $154.6\left(\mathrm{C}, \mathrm{C}-7 \mathrm{a}^{\prime}\right), 151.8$ (C, C-4), $151.4\left(\mathrm{CH}, \mathrm{C}-2^{\prime \prime}, \mathrm{CH}, \mathrm{C}-6^{\prime \prime}\right), 136.7$ (C, C-3"'), $112.0(\mathrm{CH}, \mathrm{C}-$ 3", CH, C-5"), $132.5\left(\mathrm{C}, \mathrm{C}-3 \mathrm{a}^{\prime}\right), 156.0\left(\mathrm{C}, \mathrm{C}-1^{\prime \prime}\right), 131.2\left(\mathrm{C}, \mathrm{C}-4^{\prime \prime \prime}\right), 128.5\left(\mathrm{CH}, \mathrm{C}-5^{\prime \prime \prime}\right), 124.8\left(\mathrm{C}, \mathrm{C}-6^{\prime}\right)$, $122.3\left(\mathrm{CH}, \mathrm{C}-5^{\prime}\right), 151.0\left(\mathrm{CH}, \mathrm{C}-6^{\prime \prime \prime}\right), 150.2\left(\mathrm{CH}, \mathrm{C}-2^{\prime \prime \prime}\right), 120.6\left(\mathrm{CH}, \mathrm{C}-4^{\prime}\right), 114.3(\mathrm{CH}, \mathrm{C}-5), 111.2(\mathrm{CH}$, C-7), $108.1\left(\mathrm{CH}, \mathrm{C}-3^{\prime}\right)$. MS m/z: $370\left(\mathrm{M}^{+}\right)$. Anal. Calcd. for $\mathrm{C}_{21} \mathrm{H}_{14} \mathrm{~N}_{4} \mathrm{OS}$ : C, 68.09; H, 3.81; N, 15.12; Found: C, 67.96; H, 3.76; N, 15.06.

$\mathrm{N}$-(4-(benzofuran-2-yl)-3-(4-chlorophenyl)thiazol-2(3H)-ylidene)-2,4-difluorophenyl amine (4m) White solid; yield: $69 \%$; mp 144-146 ${ }^{\circ} \mathrm{C}$. IR (KBr): 3053, $3041(\mathrm{Ar}-\mathrm{H}), 1618(\mathrm{C}=\mathrm{N}), 1599(\mathrm{C}=\mathrm{C}), 1272$ (C-S), 1074 (C-O-C), 1035 (C-F) cm ${ }^{-1} .{ }^{1} \mathrm{H}-\mathrm{NMR}$ (400 MHz, DMSO-d 6 ): $\delta$ 7.72-7.66 (m, 4H, ArH), 7.58 $(\mathrm{d}, J=8.6 \mathrm{~Hz}, 2 \mathrm{H}, \mathrm{ArH}), 7.46(\mathrm{~s}, 1 \mathrm{H}, \mathrm{ArH}), 7.18(\mathrm{~d}, J=8.6 \mathrm{~Hz}, 1 \mathrm{H}, \mathrm{ArH}), 6.92-6.85$ (m, 3H, ArH), 7.02 (s, 1H, ArH), 5.85 (s, 1H, ArH, Thiazole). ${ }^{13} \mathrm{C}$ NMR (100 MHz, DMSO-d $\left.d_{6}\right): \delta 161.1$ (C, C-2), $158.3(\mathrm{C}$, C-2'), 154.6 (C, C-7a'), 151.8 (C, C-4), 139.6 (C, C-1"), 154.0 (C, C-4'"), 128.0 (CH, C-3", CH, C-5"), 126.2 (C, C-4"), 119.1 (CH, C-2", CH, C-6") 132.5 (C, C-3a'), 151.6 (C, C-2"'), 133.4 (CH, C-1"'), 124.8 $\left(\mathrm{C}, \mathrm{C}-6^{\prime}\right), 122.3\left(\mathrm{CH}, \mathrm{C}-5^{\prime}\right), 128.8\left(\mathrm{CH}, \mathrm{C}-6^{\prime \prime \prime}\right), 124.6\left(\mathrm{CH}, \mathrm{C}-5^{\prime \prime \prime}\right), 119.0\left(\mathrm{CH}, \mathrm{C}-3^{\prime \prime \prime}\right), 120.6\left(\mathrm{CH}, \mathrm{C}-4^{\prime}\right)$, $114.3(\mathrm{CH}, \mathrm{C}-5), 111.2(\mathrm{CH}, \mathrm{C}-7), 108.1\left(\mathrm{CH}, \mathrm{C}-3^{\prime}\right)$. MS m/z: $440\left(\mathrm{M}^{+}+2\right), 438\left(\mathrm{M}^{+}\right)$. Anal. Calcd. for $\mathrm{C}_{23} \mathrm{H}_{13} \mathrm{ClF}_{2} \mathrm{~N}_{2} \mathrm{OS}$ : C, 62.94; H, 2.99; N, 6.38; Found: C, 62.91; H, 2.87; N, 6.35.

$\boldsymbol{N}$-(4-(benzofuran-2-yl)-3-(4-chlorophenyl)thiazol-2(3H)-ylidene)-2,4-dichlorophenyl amine (4n)

White solid; yield: $66 \%$; mp 148-151 ${ }^{\circ} \mathrm{C}$. IR (KBr): 3048, $3032(\mathrm{Ar}-\mathrm{H}), 1622(\mathrm{C}=\mathrm{N}), 1603(\mathrm{C}=\mathrm{C}), 1269$ (C-S), 1081 (C-O-C), 757 (C-Cl) cm ${ }^{-1} .{ }^{1} \mathrm{H}-\mathrm{NMR}$ (400 MHz, DMSO-d $)$ : $\delta$ 7.72-7.66 (m, 4H, ArH), 7.52 $(\mathrm{d}, J=8.5 \mathrm{~Hz}, 2 \mathrm{H}, \mathrm{ArH}), 7.44(\mathrm{~s}, 1 \mathrm{H}, \mathrm{ArH}), 7.26(\mathrm{~d}, J=8.8 \mathrm{~Hz}, 1 \mathrm{H}, \mathrm{ArH}), 7.12(\mathrm{~d}, J=8.8 \mathrm{~Hz}, 1 \mathrm{H}, \operatorname{ArH})$, 7.00-6.88 (m, 3H, ArH), 5.84 (s, 1H, ArH, Thiazole). ${ }^{13}$ C NMR (100 MHz, DMSO- $\left.d_{6}\right): \delta 161.1$ (C, C-2), $158.3\left(\mathrm{C}, \mathrm{C}-2^{\prime}\right), 154.6$ (C, C-7a'), 151.8 (C, C-4), 139.6 (C, C-1"'), 139.5 (C, C-1'"'), 128.0 (CH, C-3", CH, C-5"), 126.2 (C, C-4"), 119.1 (CH, C-2", CH, C-6"')132.5 (C, C-3a'), 136.2 (C, C-4"'"), 134.2 (CH, C-3"'), 124.8 (C, C-6'), $122.3\left(\mathrm{CH}, \mathrm{C}-5^{\prime}\right), 133.2\left(\mathrm{CH}, \mathrm{C}-2^{\prime \prime \prime}\right), 131.2\left(\mathrm{CH}, \mathrm{C}-5^{\prime \prime \prime}\right), 128.2\left(\mathrm{CH}, \mathrm{C}-6^{\prime \prime \prime}\right), 120.6(\mathrm{CH}$, C-4'), $114.3(\mathrm{CH}, \mathrm{C}-5), 111.2(\mathrm{CH}, \mathrm{C}-7), 108.1\left(\mathrm{CH}, \mathrm{C}-3^{\prime}\right)$. MS m/z: $473\left(\mathrm{M}^{+}+2\right), 471\left(\mathrm{M}^{+}\right)$. Anal. Calcd. for $\mathrm{C}_{23} \mathrm{H}_{13} \mathrm{Cl}_{3} \mathrm{~N}_{2} \mathrm{OS}$ : C, 58.55; H, 2.78; N, 5.94; Found: C, 58.49; H, 2.73; N, 5.92. 
RASĀYAN J. Chem.

Vol. 11 | No. 2 |661 - 675 | April - June | 2018

$N$-(4-(benzofuran-2-yl)-3-(4-chlorophenyl)thiazol-2(3H)-ylidene)-3-(trifluoromethyl) phenylamine (4o)

Pale yellow solid; yield: $68 \%$; mp 131-133 ${ }^{\circ} \mathrm{C}$. IR (KBr): 3100, $3060(\mathrm{Ar}-\mathrm{H}), 1616(\mathrm{C}=\mathrm{N}), 1600(\mathrm{C}=\mathrm{C})$, 1278 (C-S), 1082 (C-O-C), 1032 (C-F) cm ${ }^{-1} .{ }^{1} \mathbf{H}-\mathbf{N M R}$ (400 MHz, DMSO-d 6 ): $\delta$ 7.72-7.64 (m, 5H, ArH), 7.52-7.46 (m, 4H, ArH), 7.20-6.88 (m, 4H, ArH), $5.84\left(\mathrm{~s}, 1 \mathrm{H}, \mathrm{ArH}\right.$, Thiazole). ${ }^{13} \mathrm{C}$ NMR (100 MHz, DMSO- $\left.d_{6}\right): \delta 161.1(\mathrm{C}, \mathrm{C}-2), 158.3\left(\mathrm{C}, \mathrm{C}-2^{\prime}\right), 154.6\left(\mathrm{C}, \mathrm{C}-7 \mathrm{a}^{\prime}\right), 151.8(\mathrm{C}, \mathrm{C}-4), 139.6\left(\mathrm{C}, \mathrm{C}-1^{\prime \prime}\right), 152.2(\mathrm{C}$, C-1"'), 128.0 (CH, C-3", CH, C-5"), 126.2 (C, C-4"), 119.1 (CH, C-2", CH, C-6")132.5 (C, C-3a'), 136.1 (C, C-4"'), $133.4\left(\mathrm{CH}, \mathrm{C}-5^{\prime \prime \prime}\right), 124.8$ (C, C-6'), 122.3 (CH, C-5'), $128.0\left(\mathrm{CH}, \mathrm{C}-6^{\prime \prime \prime}\right), 126.3\left(\mathrm{CH}, \mathrm{C}-5^{\prime \prime \prime}\right)$, 126.8 (C, C-1"'"'), 123.3 (CH, C-2'"'), 120.6 (CH, C-4'), 114.3 (CH, C-5), $111.2(\mathrm{CH}, \mathrm{C}-7), 108.1$ (CH, C$\left.3^{\prime}\right)$. MS $m / z: 472\left(\mathrm{M}^{+}+2\right), 470\left(\mathrm{M}^{+}\right)$. Anal. Calcd. for $\mathrm{C}_{24} \mathrm{H}_{14} \mathrm{ClF}_{3} \mathrm{~N}_{2} \mathrm{OS}$ : C, 61.21; H, 3.00; N, 5.95; Found: C, 61.19; H, 2.92; N, 5.91.

\section{$N$-(4-(benzofuran-2-yl)-3-(4-chlorophenyl)thiazol-2(3H)-ylidene)-4-(trifluoromethyl) phenylamine (4p)}

Yellow white solid; yield: $65 \%$; mp $152-154{ }^{\circ} \mathrm{C}$. IR (KBr): 3071, $3052(\mathrm{Ar}-\mathrm{H}), 1621(\mathrm{C}=\mathrm{N}), 1593$ $(\mathrm{C}=\mathrm{C}), 1281(\mathrm{C}-\mathrm{S}), 1077(\mathrm{C}-\mathrm{O}-\mathrm{C}), 1042(\mathrm{C}-\mathrm{F}) \mathrm{cm}^{-1} .{ }^{1} \mathbf{H}-\mathrm{NMR}\left(400 \mathrm{MHz}, \mathrm{DMSO}-d_{6}\right): \delta$ 7.82-7.66 (m, 6H, ArH), 7.54-7.43 (m, 4H, ArH), 6.94-6.86 (m, 3H, ArH,), 5.84 (s, 1H, ArH, Thiazole). ${ }^{13} \mathbf{C}$ NMR (100 MHz, DMSO- $\left.d_{6}\right): \delta 161.1(\mathrm{C}, \mathrm{C}-2), 158.3\left(\mathrm{C}, \mathrm{C}-2^{\prime}\right), 154.6$ (C, C-7a'), 151.8 (C, C-4), $139.6\left(\mathrm{C}, \mathrm{C}-1^{\prime \prime}\right)$, 151.0 (C, C-1"'), 128.0 (CH, C-3", CH, C-5"), 126.2 (C, C-4"), 119.1 (CH, C-2", CH, C-6"')132.5 (C, C3a'), 131.2 (C, C-4"'), 128.4 (CH, C-3"', CH, C-5"''), 124.4 (CH, C-2"', CH, C-6"' '), 124.8 (C, C-6'), 122.3 $\left(\mathrm{CH}, \mathrm{C}-5^{\prime}\right), 126.6\left(\mathrm{C}, \mathrm{C}-1^{\prime \prime \prime \prime}\right), 120.6\left(\mathrm{CH}, \mathrm{C}-4^{\prime}\right), 114.3(\mathrm{CH}, \mathrm{C}-5), 111.2(\mathrm{CH}, \mathrm{C}-7), 108.1$ (CH, C-3'). MS m/z: $472\left(\mathrm{M}^{+}+2\right), 470\left(\mathrm{M}^{+}\right)$. Anal. Calcd. for $\mathrm{C}_{24} \mathrm{H}_{14} \mathrm{ClF}_{3} \mathrm{~N}_{2} \mathrm{OS}: \mathrm{C}, 61.21 ; \mathrm{H}, 3.00$; N, 5.95; Found: $\mathrm{C}$, $61.18 ; \mathrm{H}, 2.94 ; \mathrm{N}, 5.89$.

\section{$\mathrm{N}$-(4-(benzofuran-2-yl)-3-(4-nitrophenyl)thiazol-2(3H)-ylidene)-4-nitrobenzenamine (4q)}

Red solid; yield: $66 \%$; mp 158-161 ${ }^{\circ} \mathrm{C}$. IR (KBr): 3045, $3028(\mathrm{Ar}-\mathrm{H}), 1616(\mathrm{C}=\mathrm{N}), 1581(\mathrm{C}=\mathrm{C}), 1524$, $1359\left(\mathrm{NO}_{2}\right), 1272(\mathrm{C}-\mathrm{S}), 1096(\mathrm{C}-\mathrm{O}) \mathrm{cm}^{-1} .{ }^{1} \mathbf{H}-\mathrm{NMR}\left(400 \mathrm{MHz}, \mathrm{DMSO}-d_{6}\right): \delta 8.32(\mathrm{~d}, J=8.8 \mathrm{~Hz}, 2 \mathrm{H}$, $\left.\mathrm{ArH}, \mathrm{C}-3,5,4 \mathrm{NO}_{2}\right), 8.08\left(\mathrm{~d}, J=8.6 \mathrm{~Hz}, 2 \mathrm{H}, \mathrm{ArH}, \mathrm{C}-3,5,4 \mathrm{NO}_{2}\right.$ on N-thiazole), 7.72-7.66 (m, $6 \mathrm{H}, \mathrm{ArH}$, Benzofuran), 7.14 (d, $J=8.6 \mathrm{~Hz}, 2 \mathrm{H}, \mathrm{ArH}, \mathrm{C}-2,6-4 \mathrm{NO}_{2}$ on N-thiazole), 7.02 (m, 1H, ArH, Benzofuran), 5.85 (s, 1H, ArH, Thiazole). ${ }^{13}$ C NMR (100 MHz, DMSO-d $\left.d_{6}\right): \delta 161.1$ (C, C-2), 158.3 (C, C-2'), 156.8 (C, C-1"'), 154.6 (C, C-7a'), 151.8 (C, C-4), 139.3 (C, C-4"), 125.3 (C, C-3"', C-5"'), 132.5 (C, C-3a'), $122.8\left(\mathrm{CH}, \mathrm{C}-3^{\prime \prime}, \mathrm{CH}, \mathrm{C}-5^{\prime \prime}\right), 119.1\left(\mathrm{CH}, \mathrm{C}-2^{\prime \prime}, \mathrm{CH}, \mathrm{C}-6^{\prime \prime}\right), 148.3\left(\mathrm{CH}, \mathrm{C}-4^{\prime \prime \prime}\right), 124.8\left(\mathrm{C}, \mathrm{C}-6^{\prime}\right), 128.6(\mathrm{CH}$, C-2"', CH, C-6"'), $122.3\left(\mathrm{CH}, \mathrm{C}-5^{\prime}\right), 146.9\left(\mathrm{C}, \mathrm{C}-4^{\prime \prime}\right), 120.6\left(\mathrm{CH}, \mathrm{C}-4^{\prime}\right), 114.3(\mathrm{CH}, \mathrm{C}-5), 111.2(\mathrm{CH}, \mathrm{C}-$ 7), $108.1\left(\mathrm{CH}, \mathrm{C}-3^{\prime}\right)$. MS m/z: $458\left(\mathrm{M}^{+}\right)$. Anal. Calcd. for $\mathrm{C}_{23} \mathrm{H}_{14} \mathrm{~N}_{4} \mathrm{O}_{5} \mathrm{~S}: \mathrm{C}, 60.26 ; \mathrm{H}, 3.08 ; \mathrm{N}, 12.22$; Found: C, 60.20; H, 3.04; N, 12.18 .

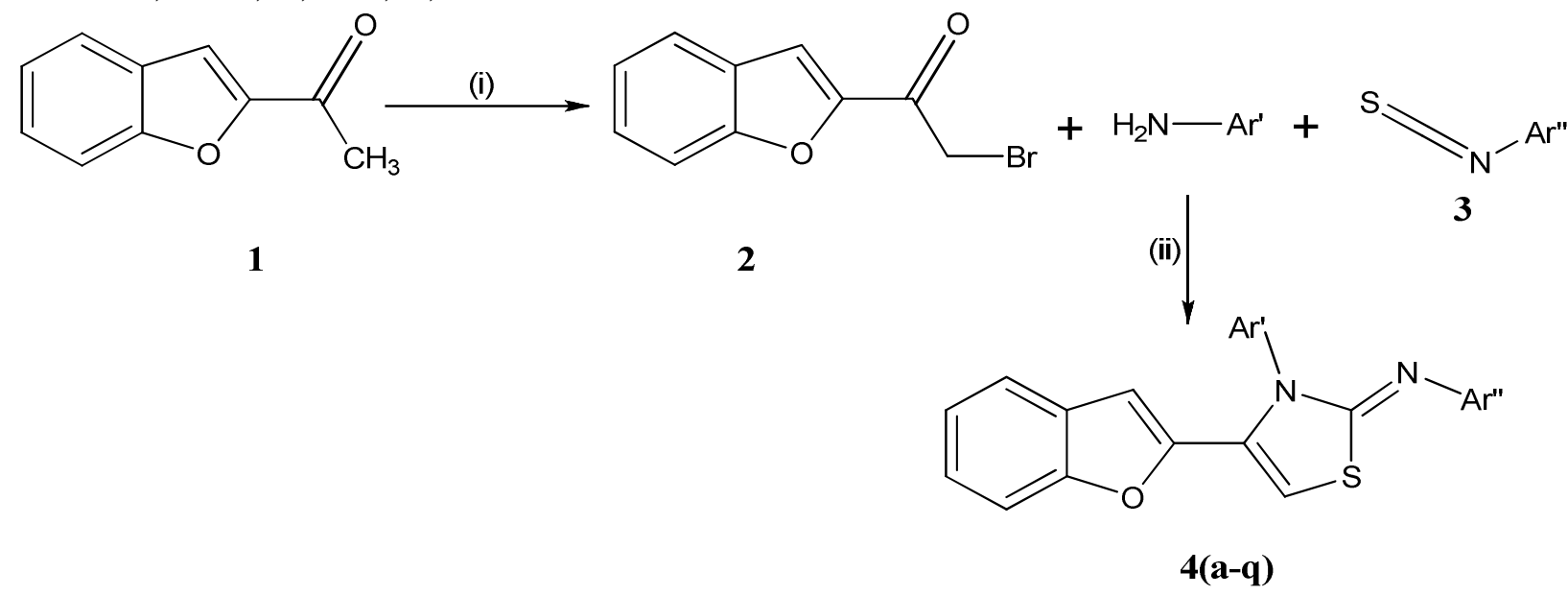

Scheme-1: Reagents and conditions: (i) $\mathrm{Br}_{2} / \mathrm{AcOH}$, reflux 90 min, 80\%; (ii) Ethanol, reflux 2 h, 64-78\%. Synthesis of N-(4-(benzofuran-2-yl)-3-arylthiazol-2(3H)-ylidene)-aryl amines 
RASĀYAN J. Chem.

Vol. 11 | No. 2 |661 - 675 | April - June | 2018

\section{Mechanism}

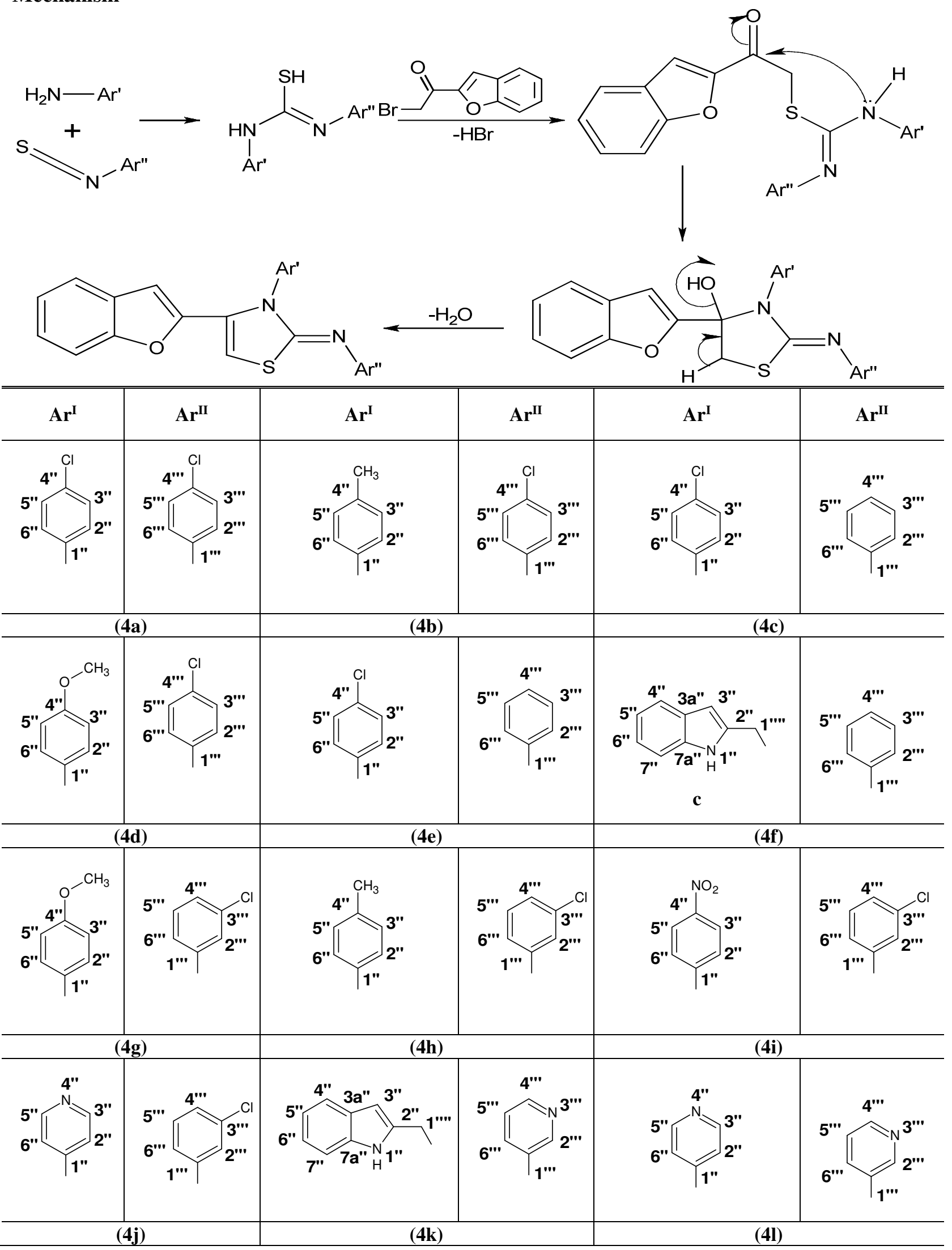


RASĀYAN J. Chem.

Vol. 11 | No. 2 |661 - 675 | April - June | 2018

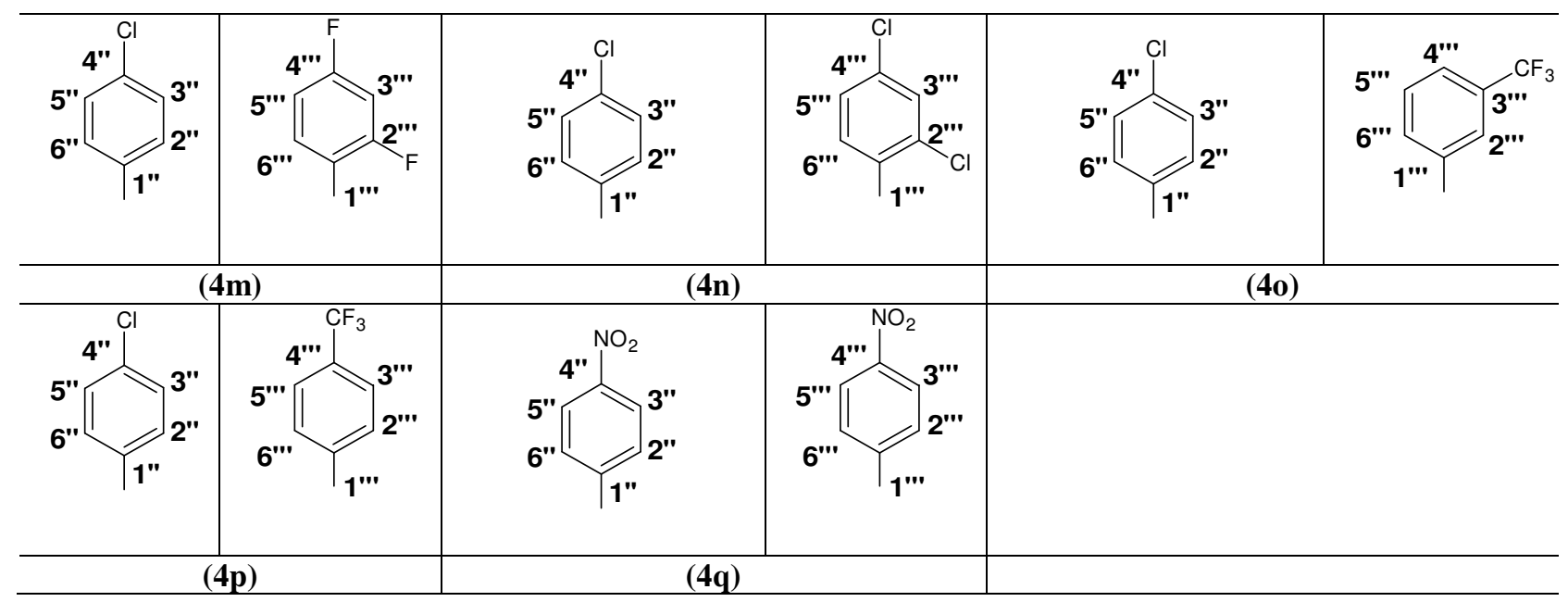

In the vitro antioxidant assay

All the final molecules $\mathbf{4}(\mathbf{a}-\mathbf{q})$ were tested antioxidant activity for their in vitro, such as nitric oxide, hydrogen peroxide free radicals scavenging activities and lipid peroxidation inhibitory activity and reducing the capacity of the compounds. The investigation of antioxidant screening revealed (Table-1) that all the newly synthesized compounds showed potent to moderate radical scavenging activity when compared to standard ascorbic acid.

\section{Cytotoxicity against cancer two cell lines}

Cytotoxicity of synthesized compounds 4(a-q) was treated with two cell lines like A549 and MDAMB231(human lung cancer \& breast cancer cell line by MTT method. All the experiments were carried out in triplicates. These two cell lines were seeded to flat bottom 96 well plates $(10,000$ cells / 100 $\mu \mathrm{L})$ and cultured in the medium having serum (10\%) incubated for $24 \mathrm{hrs}$ in a $5 \% \mathrm{Co}_{2}$ humid chamber so that the cells adhere to the surface.

MTT was dissolved in PBS at $5 \mathrm{mg} / \mathrm{mL}$ and sterile filtered. Different concentrations of the compounds were added to the adhered walls. After $48 \mathrm{~h}$ MTT sol $(10 \mu \mathrm{L}$ per well) was added to the culture plate. Cells were further incubated in the $\mathrm{CO}_{2}$ chamber for $2 \mathrm{~h}$. Following this media was removed and $100 \mu \mathrm{L}$ of DMSO was added and measured the Absorbance at $562 \mathrm{~nm}$ in a multimode micro plate recorder.

\section{RESULTS AND DISCUSSION}

We design and synthesis of the new series, $N$-(4-(benzofuran-2-yl)-3-arylthiazol-2(3H)ylidene)arylamines, as outlined in Scheme-1, commenced by bromination of commercially available 2acylbenzofuran with bromine in acetic acid and stirred for 90 min resulted in 2-bromo acetylbenzofuran 2 , with $76 \%$ yield. The compound $\mathbf{2}$, on one-pot three-component condensation reaction with aryl/ heterylamines and arylthioisocyanates in ethanol in under reflux gave the title compounds, $N-(4-$ (benzofuran-2-yl)-3-arylthiazol-2(3H)-ylidene)aryl amines 4, in good yields (85-92\%) in a short duration of about 30-40 min. All the structures of final molecules were confirmed on the basis of their spectral studies like IR, ${ }^{1} \mathrm{H}-\mathrm{NMR},{ }^{13} \mathrm{C}-\mathrm{NMR}$, mass and elemental analysis. The compounds $\mathbf{3}$ shows absorption band at about $1701 \mathrm{~cm}^{-1}$ for $\mathrm{C}=\mathrm{O}$ group which band was disappeared, but the absorption bands appeared at about 3423 and $1622 \mathrm{~cm}^{-1}$ corresponding to $\mathrm{NH}$ and $\mathrm{C}=\mathrm{N}$ stretching respectively, confirmed the formation of benzimidazole ring 4. In the ${ }^{1} \mathrm{H}$ NMR spectra, the $\mathrm{NH}$ proton of imidazole ring appeared as a singlet at about $\delta$ 9.65. In addition, aliphatic proton signals at about $\delta$ 3.90, 2.97 and 1.59-2.10, confirming the structures of the title compounds. For all the compounds the signals corresponding to the carbons of thiazole appeared at about $110.3,153.0$ and $166.8 \mathrm{ppm}$, signals corresponding to the carbons of spiro thiazolidinone appeared at about 56.2, 60.8 and $173.4 \mathrm{ppm}$ and signals corresponding to the 
carbons of benzimidazole appeared at about 118.5, 122.6, 138.4 and $151.6 \mathrm{ppm}$. All the synthesized molecules were matched with spectral data steady with their structures proposed for compounds $\mathbf{4}(\mathbf{a}-\mathbf{q})$.

\section{Biological studies \\ In vitro antioxidant activity}

Antioxidant activity was evaluated in $\mathrm{IC}_{50}$ in $\mu \mathrm{M}$ (the effective concentration at which $50 \%$ of the radicals were scavenged). The data (Table-1) revealed that compounds 4d, 4-methoxyphenyl and 4- chlorophenyl , 4l, 4-pyridyl and 3-pyridyl, 4o, 4-chlorophenyl and 3-(trifluoromethyl) phenyl and 4q, 4-nitrophenyl and 4-nitrophenyl showed higher activity than the standard antioxidant ascorbic acid. Remaining compounds showed moderate to good activity.

Table-1: Antioxidant activity of the newly synthesized compound

\begin{tabular}{c|c|c|c}
\hline \multirow{2}{*}{ Compound } & \multicolumn{3}{|c}{$\mathrm{IC}_{50} \pm$ S.D* $(\mu \mathrm{g} / \mathrm{mL})$} \\
\cline { 2 - 4 } & Nitric oxide & $\begin{array}{c}\text { Hydrogen } \\
\text { peroxide }\end{array}$ & Lipid peroxidation \\
\hline $4 \mathrm{a}$ & $61 \pm 0.35$ & $42 \pm 0.26$ & $52 \pm 0.19$ \\
\hline $4 \mathrm{~b}$ & $<100$ & $61 \pm 1.18$ & $77 \pm 1.44$ \\
\hline $4 \mathrm{c}$ & $42 \pm 0.35$ & $72 \pm 0.35$ & $<100$ \\
\hline $4 \mathrm{~d}$ & $40 \pm 0.32$ & $44 \pm 0.26$ & $41 \pm 0.16$ \\
\hline $4 \mathrm{e}$ & $54 \pm 0.15$ & $68 \pm 0.19$ & $76 \pm 0.24$ \\
\hline $4 \mathrm{f}$ & $75 \pm 2.16$ & $66 \pm 1.25$ & $32 \pm 1.28$ \\
\hline $4 \mathrm{~g}$ & $52 \pm 0.25$ & $<100$ & $80 \pm 1.24$ \\
\hline $4 \mathrm{~h}$ & $58 \pm 1.18$ & $61 \pm 1.11$ & $64 \pm 1.42$ \\
\hline $4 \mathrm{i}$ & $56 \pm 0.79$ & $63 \pm 0.87$ & $79 \pm 0.27$ \\
\hline $4 \mathrm{j}$ & $68 \pm 1.68$ & $<100$ & $66 \pm 1.21$ \\
\hline $4 \mathrm{k}$ & $<100$ & $71 \pm 0.87$ & $34 \pm 0.23$ \\
\hline 41 & $32 \pm 0.42$ & $36 \pm 0.34$ & $54 \pm 0.59$ \\
\hline $4 \mathrm{~m}$ & $42 \pm 0.27$ & $39 \pm 0.38$ & $91 \pm 0.38$ \\
\hline $4 \mathrm{n}$ & $84 \pm 0.62$ & $<100$ & $42 \pm 0.16$ \\
\hline $4 \mathrm{o}$ & $44 \pm 0.32$ & $48 \pm 0.26$ & $39 \pm 0.13$ \\
\hline $4 \mathrm{p}$ & $<100$ & $82 \pm 0.74$ & $37 \pm 0.23$ \\
\hline $4 \mathrm{q}$ & $36 \pm 0.03$ & $41 \pm 0.08$ & $38 \pm 0.71$ \\
\hline Ascorbic acid & $33 \pm 0.12$ & & \\
\hline
\end{tabular}

\section{In vitro Anticancer activity}

All compounds (4a-q) were subjected to screening for anticancer activity, against A549 (Lung cancer) and MDAMB-231 (Breast cancer) cell lines revealed that, (Table-2) compounds bearing, 4-chloro; 4chloro (4a), 4-methoxy; 4-chloro (4d), 4-pyridinyl; 3-pyridine (4l), 4-chloro; 2,4-difluoro (4m) and 4nitro; 4-nitro (4q) against A549 have shown significant activity with $\mathrm{IC}_{50}=8.00 \pm 1.21 \mu \mathrm{g} / \mathrm{mL}, \mathrm{IC}_{50}=$ $4.12 \pm 1.51 \mu \mathrm{g} / \mathrm{mL}, \mathrm{IC}_{50}=6.21 \pm 0.37 \mu \mathrm{g} / \mathrm{mL}, 7.33 \pm 0.54 \mu \mathrm{g} / \mathrm{mL}$ and $\mathrm{IC}_{50}=7.80 \pm 0.57 \mu \mathrm{g} / \mathrm{mL}$ respectively. Similarly, compounds bearing, 4-methyl; phenyl (4e), 4-nitro; 3-chloro (4i), 4-pyridinyl; 3pyridine (4I), 4-chloro; 3-trifluoro (4o) and 4-nitro; 4-nitro (4q) exhibited prominent activity against MDAMB-231 cell line with $\mathrm{IC}_{50}=4.11 \pm 0.59 \mu \mathrm{g} / \mathrm{mL}, \mathrm{IC}_{50}=2.43 \pm 1.12 \mu \mathrm{g} / \mathrm{mL}, \mathrm{IC}_{50}=3.80 \pm 0.52$ $\mu \mathrm{g} / \mathrm{mL}, \mathrm{IC}_{50}=3.73 \pm 0.35 \mu \mathrm{g} / \mathrm{mL}$ and $\mathrm{IC}_{50}=4.90 \pm 1.64 \mu \mathrm{g} / \mathrm{mL}$ respectively. Compounds $\mathbf{4 l}$ and $\mathbf{4 q}$ are promising compounds to sustain at low percentage of $\mathrm{IC}_{50}$ and revealed superior potency than standard doxorubicin supported by both antioxidant and docking studies as well. All most all compounds demonstrated reasonable to good inhibitory effects beside the human cancer cells at the tested concentrations comparable to doxorubicin.

Table-2: Anticancer activity of newly synthesized compounds 4(a-q)

\begin{tabular}{c|c|c}
\hline & \multicolumn{2}{|c}{$\mathrm{IC}_{50}(\mu \mathrm{g} / \mathrm{mL})$} \\
\hline Compound & $\mathrm{A} 549$ & MDAMB-231 \\
\hline $4 \mathrm{a}$ & $8.00 \pm 1.21$ & $61.00 \pm 0.88$ \\
\hline
\end{tabular}


RASĀYAN J. Chem.

Vol. 11 | No. 2 |661 - 675 | April - June | 2018

\begin{tabular}{c|c|c}
\hline $4 \mathrm{~b}$ & $66.10 \pm 1.72$ & $>100$ \\
\hline $4 \mathrm{c}$ & $48.20 \pm 0.36$ & $30.23 \pm 1.62$ \\
\hline $4 \mathrm{~d}$ & $4.12 \pm 1.51$ & $12.33 \pm 3.36$ \\
\hline $4 \mathrm{e}$ & $25.60 \pm 0.22$ & $4.11 \pm 0.59$ \\
\hline $4 \mathrm{f}$ & $33.16 \pm 0.94$ & $25.40 \pm 2.88$ \\
\hline $4 \mathrm{~g}$ & $78.53 \pm 5.76$ & $>100$ \\
\hline $4 \mathrm{~h}$ & $13.18 \pm 1.34$ & $2.43 \pm 1.12$ \\
\hline $4 \mathrm{i}$ & $18.00 \pm 4.21$ & $38.10 \pm 1.74$ \\
\hline $4 \mathrm{j}$ & $83.24 \pm 2.66$ & $72.40 \pm 2.12$ \\
\hline $4 \mathrm{k}$ & $54.11 \pm 0.18$ & $3.80 \pm 0.52$ \\
\hline $4 \mathrm{l}$ & $6.21 \pm 0.37$ & $22.10 \pm 2.92$ \\
\hline $4 \mathrm{~m}$ & $7.33 \pm 0.54$ & 3100 \\
\hline $4 \mathrm{n}$ & $>100$ & $86.32 \pm 2.37$ \\
\hline $4 \mathrm{o}$ & $72.19 \pm 4.56$ & $4.90 \pm 1.64$ \\
\hline $4 \mathrm{p}$ & $>100$ & $5.96 \pm 0.13$ \\
\hline $4 \mathrm{q}$ & $7.8 \pm 0.57$ & 0.35 \\
\hline Doxorubicin & $8.05 \pm 0.37$ & $\mathrm{~s}$ \\
\hline
\end{tabular}

Data are expressed as means \pm SEM of two independent experiments performed in triplicates

\section{Molecular Docking Analysis}

Docking studies are computer-based drug design for biological evaluation continues to hold pronounced assurance in the field, to avoid riskier human trials and to reduce animal testing of synthesized compounds. Certainly, these studies open the door to unfold the biological processes without compromising the accuracy, providing new insights used to evaluate biological properties of the molecule in further stages of analysis at the atomic level. In this framework, in silico molecular docking investigation merits are understood the essential role of the benzofuran derivatives $\mathbf{4}(\mathbf{a}-\mathbf{q})$ as anticancer agents. To attain this assignment, compounds are oriented in the active sphere of targeted proteins retrieved from PDB site with ID: 2ITO (EGFR kinase) and 3EQM (Aromatase) and their aptness in regulating the functions were studied from their binding interaction energies, calculated using the scoring functions.

\section{Anti-lung cancer - docking Protein ID: 2ITO}

Analysis of the binding patterns and their docking score between Chrysin and each of synthesized compounds against target protein EGFR kinase with protein ID: 2ITO (Table-3) revealed that, compounds 4d and $\mathbf{4 l}$ showed the best fitness than other compounds. Visual inspection of docked structures of $\mathbf{4 d}$ and $4 \mathbf{l}$ with the receptor (Fig.-1 and 2) showed many common hydrophobic interactions with residues Cys797, Leu718, Pro794, Met793, Leu792, Ala743, Val726, Leu844, Leu788 and Met766. These are almost common in compounds $4 \mathrm{a}-\mathrm{q}$ and considered as the key active interactions responsible for varied binding affinities in receptor protein. Thus, compounds $\mathbf{4 d}$ and $\mathbf{4 l}$ are a promising candidate for further investigation as potential anti-lung cancer agents.

\section{Anti-Breast Cancer-docking \\ Protein ID: 3EQM}

The theoretical outcome of the binding patterns and their binding free energies between chrysin and each of synthesized compounds against target protein aromatase with protein ID: 3EQM (Table-4) depicted that, compounds $\mathbf{4 l}$ and $\mathbf{4 0}$ showed high binding affinities (Fig.-4) than other compounds.In order to define the key binding interactions, where specific roles of each individual pharmacophore of compounds 40 and $\mathbf{4 l}$ in the active pocket of target protein were explored and found the conserved hydrophobic 
RASĀYAN J. Chem.

Vol. 11 | No. 2 |661-675 | April - June | 2018

interactions with residues Cys437, Ile133, Ala438, Ala306, Ala307, Met446, Leu152, Phe430, Met311, Ala443 and Val370.
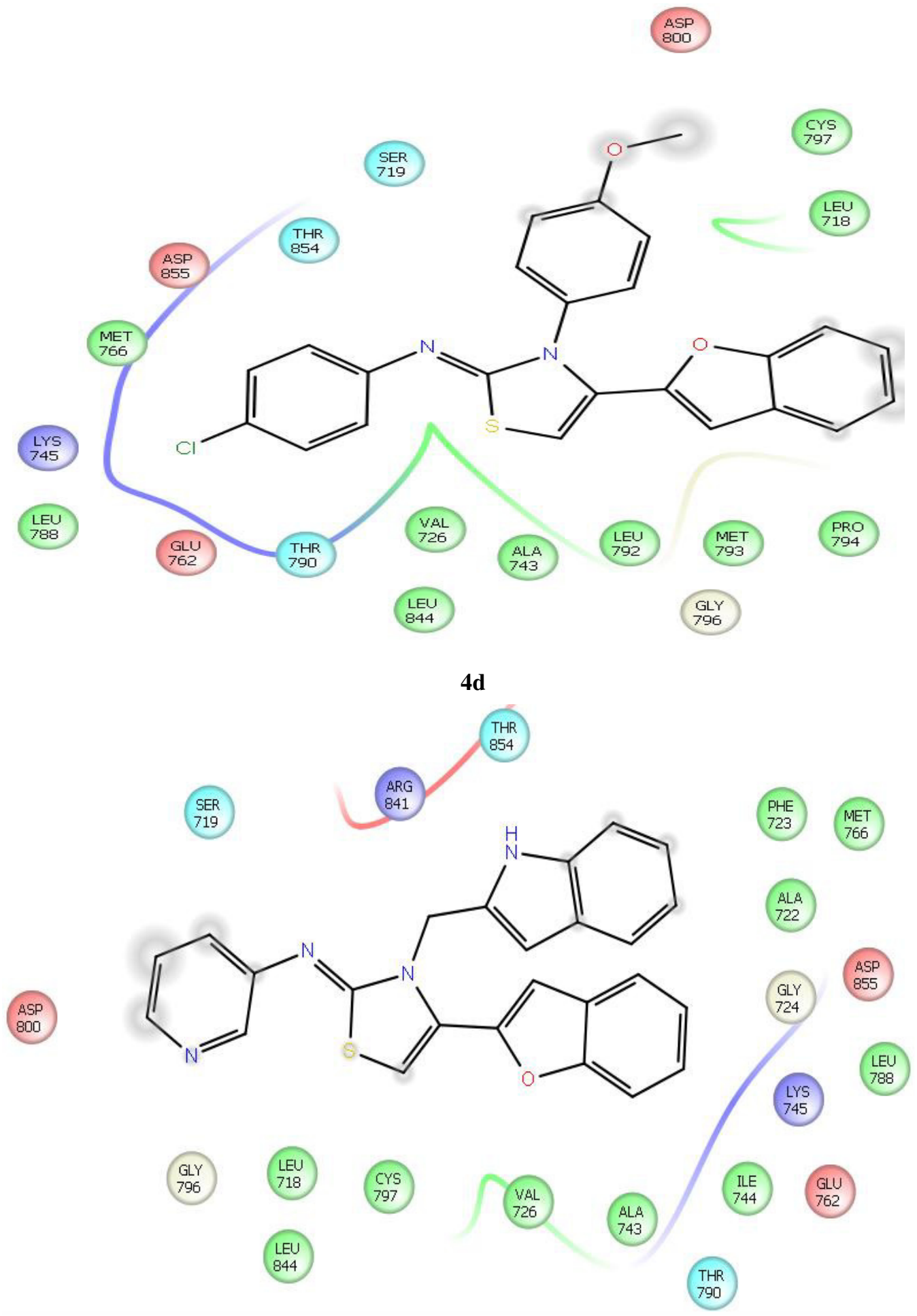

41

Fig.-1: Compounds $\mathbf{4 d}$ and $\mathbf{4 l}$ showed the best fitness than other compounds with many common hydrophobic interactions with residues Cys797, Leu718, Pro794, Met793, Leu792, Ala743, Val726, Leu844, Leu788 and Met766. 
RASĀYAN J. Chem.

Vol. 11 | No. 2 |661-675 | April - June | 2018
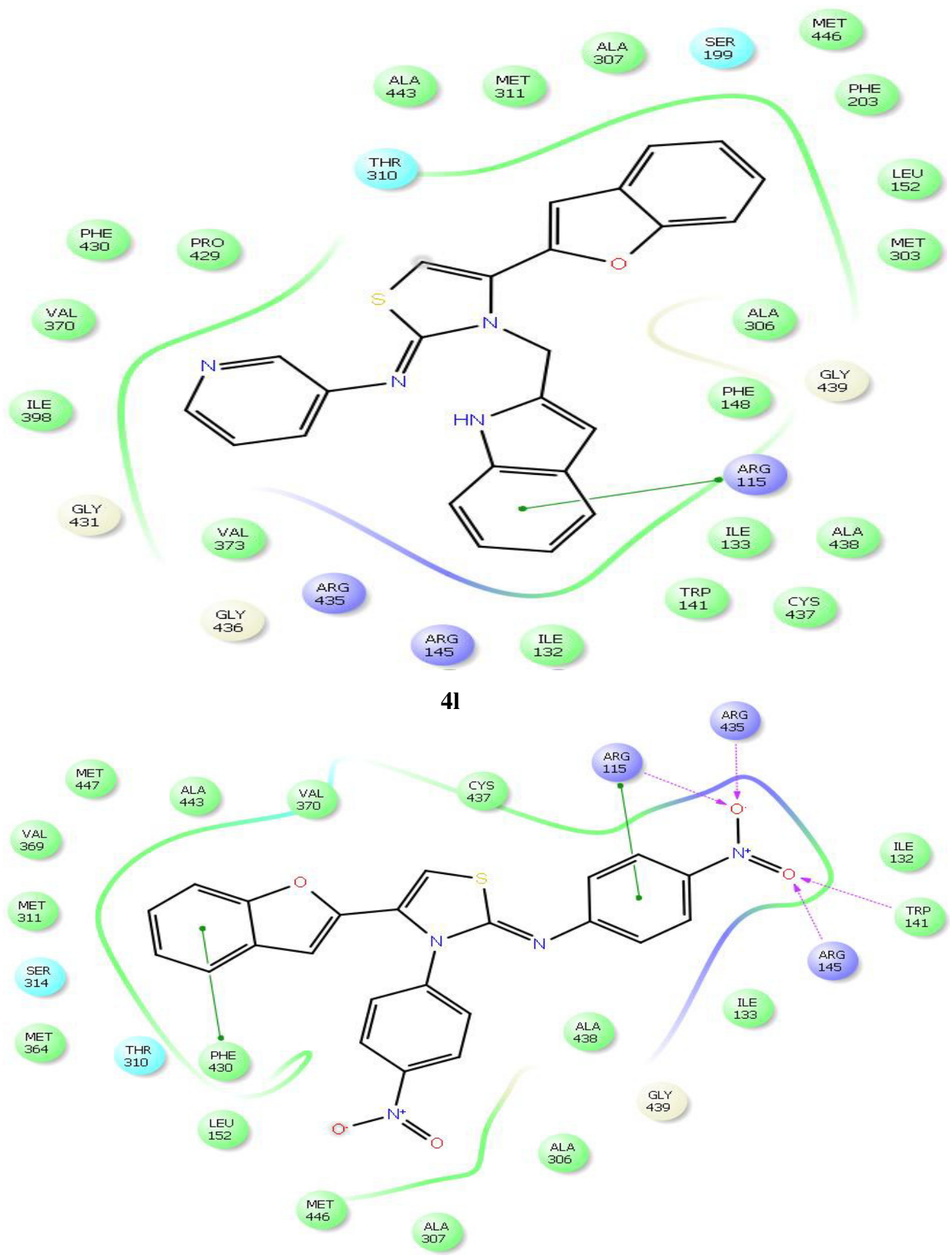

Fig.-2: Compounds 41 and 4o showed high bi9ding affinities than other compounds with conserved hydrophobic interactions with residues Cys437, Ile133, Ala438, Ala306, Ala307, Met446, Leu152, Phe430, Met311, Ala443 and Val370. In addition to this, 4o showed H-bonding with Arg115, Arg435 and Arg145, among which Arg115 also depicted $\pi-\pi$ interaction.

Table -3: Anticancer (2ITO) docking of newly synthesized compounds

\begin{tabular}{|c|c|c|c|c|c|c|}
\hline \multirow[b]{2}{*}{ Compound } & \multicolumn{3}{|c|}{ H-bond } & \multirow[b]{2}{*}{ Residue ID } & \multirow[b]{2}{*}{$\begin{array}{c}\text { Docking } \\
\text { Score }\end{array}$} & \multirow[b]{2}{*}{ Rank } \\
\hline & $\begin{array}{l}\text { Atom/ group of } \\
\text { compound }\end{array}$ & Nature & $\begin{array}{c}\text { Distance } \\
\left(\mathrm{A}^{0}\right)\end{array}$ & & & \\
\hline $4 a$ & - & - & - & - & 7.166 & 4 \\
\hline
\end{tabular}


RASĀYAN J. Chem.

Vol. 11 | No. 2 |661 - 675 | April - June | 2018

\begin{tabular}{|c|c|c|c|c|c|c|}
\hline $4 b$ & - & - & - & - & 6.268 & 12 \\
\hline $4 \mathrm{c}$ & - & - & - & - & 6.638 & 10 \\
\hline $4 d$ & - & - & - & - & 7.245 & 1 \\
\hline $4 \mathrm{e}$ & - & - & - & - & 6.721 & 8 \\
\hline $4 f$ & $\begin{array}{c}\text {-NH of } \\
\text { imidazole }\end{array}$ & H-donor & 1.90 & $\begin{array}{c}-\mathrm{COO}^{-} \text {of } \\
\text { Asp855 }\end{array}$ & 6.657 & 9 \\
\hline $4 g$ & - & - & - & - & 6.134 & 15 \\
\hline $4 \mathrm{~h}$ & - & - & - & - & 7.062 & 6 \\
\hline $4 \mathrm{i}$ & 一 & - & - & - & 6.934 & 7 \\
\hline $4 j$ & - & - & - & - & 6.158 & 14 \\
\hline $4 \mathrm{k}$ & - & - & - & - & 6.297 & 11 \\
\hline 41 & - & - & - & - & 7.177 & 2 \\
\hline $4 \mathrm{~m}$ & - & - & - & - & 7.167 & 3 \\
\hline $4 n$ & - & - & - & - & 5.271 & 17 \\
\hline 40 & $-\mathrm{NO}_{2}$ of phenyl & H-acceptor & 1.84 & $\begin{array}{l}-\mathrm{NH}_{2} \text { of } \\
\text { Met793 }\end{array}$ & 6.239 & 13 \\
\hline $4 p$ & 一 & - & - & - & 5.618 & 16 \\
\hline $4 q$ & - & - & - & - & 7.083 & 5 \\
\hline
\end{tabular}

In addition to this, 4o showed H-bonding with Arg115, Arg435 and Arg145, among which Arg115 also depicted $\pi-\pi$ interaction (Fig.-4). These binding interactions and H-bonding in the active pocket of targeted protein reveal that, H-bonds and $\pi-\pi$ interactions with Ala115 are key binding sites to regulate the action of the protein. Thus, compounds $\mathbf{4 l}$ and $\mathbf{4 o}$ are a promising candidate for further investigation as a potential anti-breast cancer drugs.

Table-4: Anticancer (3EQM) docking of newly synthesized compounds

\begin{tabular}{|c|c|c|c|c|c|c|}
\hline \multirow[b]{2}{*}{ Compound } & \multicolumn{3}{|c|}{ H-bond } & \multirow[b]{2}{*}{ Residue ID } & \multirow{2}{*}{$\begin{array}{c}\text { Docking } \\
\text { Score }\end{array}$} & \multirow[b]{2}{*}{ Rank } \\
\hline & $\begin{array}{l}\text { Atom/ group of } \\
\text { compound }\end{array}$ & Nature & $\begin{array}{l}\text { Distance } \\
\left(\mathrm{A}^{0}\right)\end{array}$ & & & \\
\hline $4 a$ & - & - & - & - & 6.505 & 10 \\
\hline $4 b$ & - & - & - & - & 6.230 & 13 \\
\hline $4 c$ & - & - & - & - & 6.582 & 9 \\
\hline $4 d$ & - & - & - & - & 5.997 & 15 \\
\hline $4 \mathrm{e}$ & - & - & - & - & 6.857 & 5 \\
\hline $4 \mathrm{f}$ & - & - & - & - & 6.656 & 8 \\
\hline $4 g$ & - & - & - & - & 5.503 & 17 \\
\hline $4 \mathrm{~h}$ & - & - & - & - & 6.763 & 6 \\
\hline $4 \mathrm{i}$ & - & - & - & - & 6.859 & 4 \\
\hline $4 \mathrm{j}$ & - & - & - & - & 6.062 & 14 \\
\hline $4 \mathrm{k}$ & - & - & - & - & 6.477 & 11 \\
\hline 41 & - & - & - & - & 6.964 & 2 \\
\hline $4 \mathrm{~m}$ & - & - & - & - & 6.703 & 7 \\
\hline $4 n$ & - & - & - & - & 5.686 & 16 \\
\hline \multirow[t]{4}{*}{40} & $-\mathrm{NO}_{2}$ of phenyl & $\mathrm{H}$-acceptor & 2.20 & $\begin{array}{l}-\mathrm{NH}_{3} \text { of } \\
\text { Arg115 }\end{array}$ & 7.470 & 1 \\
\hline & $-\mathrm{NO}_{2}$ of phenyl & H-acceptor & 2.44 & $\begin{array}{c}=\mathrm{NH}_{2}{ }^{+} \text {of } \\
\text { Arg435 }\end{array}$ & & \\
\hline & $-\mathrm{NO}_{2}$ of phenyl & H-donor & 2.21 & $\begin{array}{l}-\mathrm{NH}_{2} \text { of } \\
\text { Arg145 }\end{array}$ & & \\
\hline & $-\mathrm{NO}_{2}$ of phenyl & H-donor & 1.79 & $\begin{array}{l}\text {-NH of } \\
\text { Trp141 }\end{array}$ & & \\
\hline $4 p$ & - & - & - & - & 6.315 & 12 \\
\hline $4 q$ & - & 一 & - & - & 6.956 & 3 \\
\hline
\end{tabular}


RASĀYAN J. Chem.

Vol. 11 | No. 2 |661-675 | April - June | 2018

\section{CONCLUSION}

A series of Some New $N$-(4-(benzofuran-2-yl)-3-arylthiazol-2(3H)-ylidene)-arylamines 4a-q were synthesized and characterized by their spectral data and elemental analyses. Antioxidant activity revealed that the compounds $\mathbf{4 d}, \mathbf{4}, \mathbf{4} \mathbf{o}$ and $\mathbf{4 q}$ emerged as better antioxidants than the standard. In-vitro anticancer activity reveals that, compounds $\mathbf{4 a}, \mathbf{4 d}, \mathbf{4 l}$ and $\mathbf{4 q}$ emerged as potent anti-lung cancer agents and compounds $\mathbf{4 e}, \mathbf{4 i}, \mathbf{4 l}$ and $\mathbf{4 q}$ evolved as promising anti-breast cancer drugs with superior potency than doxorubicin. Further the molecular docking exploration of binding interactions unveils the best fitness and potent inhibiting effect on the EGFR kinase and aromatase respectively by virtue of their strong binding affinity with the amino acid residues of the enzymes. Most of the newly synthesized compounds explicit appreciable activity against tested cell lines. These capable results are consistent, however, subjected to preclinical studies to get there at the termination of these molecules for their clinical use and may come out as influential anticancer agents.

\section{ACKNOWLEDGMENT}

Authors are thankful to Chaitanya Colleges, Warangal for providing facilities to carry out the research work, Dr. T. Balraj, Indian Institute of Science Education and Research, Kolkata for his support in molecular docking analysis and financial assistance for research grants from the UGC, SERO, Hyderabad, India, is greatly acknowledged.

1. O. C. R. Sorg, Biologies., 327, 649 (2004).

2. K. J. Davies, Biochem. Soc. Symp., 61, 1(1995).

3. B. N. Ames, Science., 221, 1256 (1983).

4. J. A. Berliner and J. W. Heinecke., Free Radic. Biol. Med., 20(5), 707(1996).

5. X. Tang, H. Shigematsu, B. N. Bekele, J. A. Roth, J. D. Minna, W. K. Hong, A. F. Gazdar, I.I. Wistuba, Cancer Res., 65(17), 7568 (2005). DOI: 10.1158/0008-5472. CAN-05-1705

6. P. Traxler, G. Bold, E. Buchdunger, G. Caravatti, P. Furet, P. Manley, T. O'Reilly, J. Wood, J. Zimmermann, Med. Res. Rev., 21(6), 499(2001).

7. A. Eisen, M. Trudeau, W. Shelley, H. Messersmith, K. I. Pritchard, Cancer Treat. Rev., 34 (2), 157 (2008). DOI: 10.1016/j.ctrv.2007.11.001

8. R.G. Frazen, J. Comb. Chem., 2, 195 (2000). DOI: 10.1021/cc000002f

9. P.D. Leeson, B. Springthorpe, Nat. Rev. Drug Discov., 6(11), 881(2007). DOI: 10.1038/nrd2445

10. R. W. DeSimone, K. S. Currie, S. A. Mitchell, J. W. Darrow, D. A. Pippin, Comb. Chem. High. Throughput Screen., 7(3), 473 (2004). DOI: 10.2174/ 1386207043328544

11. M. Kamal, A. K. Shakya, T. Jawaid, Int. J. Med. Pharm. Sci., 1, 1 (2011).

12. C. Kirilmis, M. Ahmedzade, S. Servi, M. Koca, A. Kizirgil, Eur. J. Med. Chem., 43(2), 300 (2008). DOI: 10.1016/j.ejmech.2007.03.023

13. Y. S. Xie, D. Kumar, V. D. V. Bodduri, P. S. Tarani, B. X. Zhao, J. Y. Miao, K. Jang, D. S. Shin, Tetrahedron Lett., 55, 2796 (2014). DOI: 10.1080/14786419.2018.1428598

14. F. Xie, H. Zhu, H. Zhang, Q. Lang, L. Tang, Q. Huang, L. Yu. Eur. J. Med. Chem., 89, 310 (2015). DOI: $10.1016 /$ j.ejmech.2014.10.044

15. S. L. Patil, C. M. Bhalgat, S. Burli, S. K. Chithale. J. Chem. Sci. \& Appl., 1, 42 (2010).

16. N. C. Desai, N. Bhatt, H. Somani, A. Trivedi, Eur. J. Med. Chem., 67, 54 (2013). DOI: 10.1016/j.ejmech.2013.06.029

17. P. K. Deb, R. Kaur, B. Chandrasekaran, M. Bala, D. Gill, V. R. Kaki, R. R. Akkinepalli, R. Mailavaram, Med. Chem. Res., 23, 2780 (2014). DOI: 10.1007/s00044-013-0861-4

18. M. H. M. Helal, M. A. Salem, M. S. A. El-Gaby, M. Aljahdali, Eur. J. Med. Chem., 65, 517 (2013). DOI: $10.1016 / j$.ejmech.2013.04.005

19. K. M. Dawood and S. M. Gomha, J. Heterocyclic Chem., 52(5), 1400 (2015). DOI: $10.1002 /$ jhet. 2250 
RASĀYAN J. Chem. Vol. 11 | No. 2 |661 - 675 | April - June | 2018

20. R. K. Rawal, R. Tripathi, S. B. Katti, C. Pannecouque, E. D. Clercq,. Eur. J. Med. Chem., 43, 2800 (2008). DOI: 10.1016/j.ejmech.2007.12.015

21. V. Jaishree, N. Ramdas, J. Sachin, B. Ramesh, J. Saudi Chem. Soc., 16, 371 (2012). DOI: 10.1016/j.jscs.2011.02.007

22. S. N. Thore, Sunil V. Gupta, K. G. Baheti, Med. Chem. Res., 22(8), 3802 (2013). DOI: 10.1007/s00044-012-0382-6

[RJC-2062/2018] 